\title{
Differentially Private Deep Learning with Iterative Gradient Descent Optimization
}

\author{
XIAOFENG DING, LIN CHEN, PAN ZHOU, WENBIN JIANG, and HAI JIN, \\ Huazhong University of Science and Technology, China
}

\begin{abstract}
Deep learning has achieved great success in various areas and its success is closely linked to the availability of massive data. But in general, a large dataset could include sensitive data and therefore the model should have the capability to avoid privacy leakage. To achieve this aim, many works apply the famous privacy framework named differential privacy into deep learning to preserve privacy. In this article, we propose a novel perturbed iterative gradient descent optimization (PIGDO) algorithm and prove that this algorithm satisfies the differential privacy. Besides, we propose a modified moments accountant (MMA) method to conduct the privacy analysis and obtain a tighter bound of privacy loss compared with the original moments accountant method. A number of experiments demonstrate that our optimization algorithm can not only improve the model accuracy and training speed, but also achieve better privacy guarantees over the stateof-the-art algorithm while reaching the equivalent accuracy. We provide codes for all of our experiments in https://github.com/CGCL-codes/DPDLIGDO.git.
\end{abstract}

CCS Concepts: • Security and privacy $\rightarrow$ Privacy-preserving protocols; $\bullet$ Computing methodologies $\rightarrow$ Neural networks;

Additional Key Words and Phrases: Differential privacy, deep learning, gradient descent optimization, privacy loss

\section{ACM Reference format:}

Xiaofeng Ding, Lin Chen, Pan Zhou, Wenbin Jiang, and Hai Jin. 2022. Differentially Private Deep Learning with Iterative Gradient Descent Optimization. ACM/IMS Trans. Data Sci. 2, 4, Article 34 (February 2022), 27 pages.

https://doi.org/10.1145/3491254

This work was supported in part by the National Natural Science Foundation of China under Grant 62172179, Grant 61972448, and Grant 61772215 and in part by the CCF-Huawei Innovation Research Plan under Grant CCFHuaweiDBIR2021006B.

Authors' addresses: X. Ding, L. Chen (corresponding author), W. Jiang, and H. Jin, National Engineering Research Center for Big Data Technology and System Lab, Services Computing Technology and System Lab, Cluster and Grid Computing Lab, School of Computer Science and Technology, Huazhong University of Science and Technology, Wuhan, 430074, China; emails: \{xfding, chenl, wenbinjiang, hjin\}@hust.edu.cn; P. Zhou (corresponding author), Hubei Engineering Research Center on Big Data Security, School of Cyber Science and Engineering, Huazhong University of Science and Technology, Wuhan, 430074, China; email: panzhou@hust.edu.cn.

Permission to make digital or hard copies of all or part of this work for personal or classroom use is granted without fee provided that copies are not made or distributed for profit or commercial advantage and that copies bear this notice and the full citation on the first page. Copyrights for components of this work owned by others than ACM must be honored. Abstracting with credit is permitted. To copy otherwise, or republish, to post on servers or to redistribute to lists, requires prior specific permission and/or a fee. Request permissions from permissions@acm.org.

(C) 2022 Association for Computing Machinery.

2577-3224/2022/02-ART34 \$15.00

https://doi.org/10.1145/3491254 


\section{INTRODUCTION}

Recently, deep learning has become more pervasive and played a significant role in all fields of AI, such as image recognition [15, 32], natural language processing [17, 24], and speech identification $[4,5]$. These achievements are mainly attributed to the enhanced computing performance, the availability of massive data, and the breakthroughs in all kinds of deep learning algorithms.

While deep learning has acquired great success, its safety concerns have also received attention. Recent studies [11,34] have shown that a well-trained machine learning model is also vulnerable to privacy risks. For example, under membership inference attacks [34], even though an individual is anonymous, the adversary can still infer whether the individual existed in the dataset. In addition, the large datasets used for deep learning are collected from individual users and these personal data typically contain some private information, such as location information, finical situation, and medical records. But in general, the users can not decide how their data will be used and shared once it was collected by a third party. What is more, deep learning networks have many hidden layers and they possess the strong capability to encode some individual data into model parameter or even memorize the whole dataset. As has been shown in Reference [11], training data could be effectually extracted from neural networks. Therefore, it is necessary to consider privacy protection while utilizing deep learning.

Differential privacy (DP) [8], proposed by Dwork et al., is a robust privacy protection mechanism. Compared with other privacy preserving methods, differential privacy can resist various types of attacks under the condition that the adversary has the maximum background knowledge. In addition, it builds on the solid mathematical foundation to give a strict definition of privacy and to provide a quantitative evaluation method. By virtue of these advantages, differential privacy theory has gradually become a hot topic in the field of privacy protection. Recently, there have been some studies applying differential privacy to deep learning [1,33]. In Reference [33], the authors first combined differential privacy with deep learning, but their algorithm caused too much privacy loss. Abadi et al. [1] improved [33] by utilizing higher moments of the privacy loss random variable and made use of differentially private SGD algorithm that added scaled noise to each step of the stochastic gradient descent for avoiding information leakage.

Following their gradient perturbation based approaches, many works [14, 30, 41, 43] proposed all kinds of tricks to improve the accuracy of gradient perturbation while achieving the satisfying privacy preservation. The main improved methods contain two types. The first approach adopted by References $[41,43]$ investigates the sensitivity of each gradient component to add the sensitivitydependent noise for higher model accuracy. The other improvement strategy used in References $[14,30]$ adaptively injects noise into gradients based on the relevance between different features and the model output. However, for the gradient perturbation methods based on the sensitivity analysis, it is usually required to solve the high-dimensional sensitivity constrained condition, which is not easy to satisfy in the deep neural network. For the gradient perturbation approaches based on the relevance analysis, the calculation of relevance of each feature in different neural network layers results in the inefficient computation. Although these two types of methods are both well designed for adding proper noise to each gradient or the gradients of each neuron, it brings the more strict requirement to achieve the feasible and efficient privacy-preserving learning algorithm. Therefore, considering that the limitations of the above noisy gradient mechanism, we consider improving the gradient perturbation method for all the gradients from an overall perspective.

Moreover, most of the existing work in differentially private deep learning took advantage of the differentially private versions of stochastic gradient descent to control the influence of the training data in the training process. Nevertheless, the SGD algorithm has some of its own drawbacks. For 


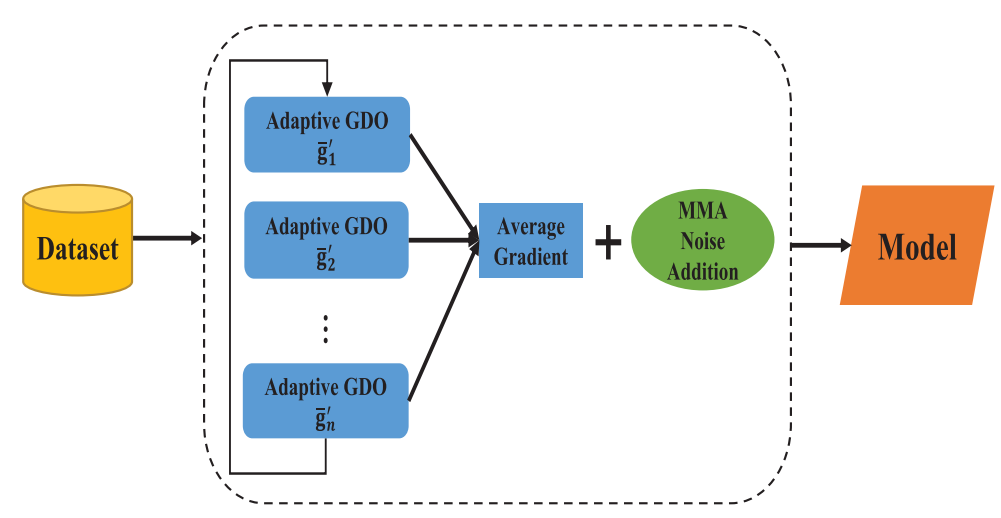

Fig. 1. The overview of PIGDO deep learning framework.

example, it has difficulty in escaping from saddle points and choosing a proper learning rate, for it is not easy. Instead, these disadvantages can be overcome by several other adaptive gradient descent optimization algorithms such as Adam [18], RMSprop [38], and Adagrad [27]. These optimization algorithms are extensively used in deep neural networks for minimizing the objective function, because they possess certain characteristics that can alleviate drawbacks of conventional SGD. In theory, these algorithms provide the better convergence rate than conventional SGD. Therefore, in this article, we choose the gradient descent optimization algorithms such as Adam algorithm to perform the gradient descent in deep learning. In the end, based on the aforementioned analysis, we integrate the gradient descent optimization algorithm as an iterative component and then inject the proper noise into this component for achieving the better overall model utility. The frame of our differentially private gradient descent optimization model is shown in Figure 1.

Our contributions can be summarized as follows:

- We present a novel perturbed iterative gradient descent optimization (PIGDO) algorithm that integrates the gradient descent algorithm as an iterative component and then adds noise to the gradients computed by iterative GDO to perform the gradient perturbation for obtaining the differential privacy. Compared with the previous noise addition mechanism designed for each gradient, our algorithm improves the accuracy of the gradients from an overall perspective and therefore achieves the better model utility while guaranteeing the privacy requirement.

- We give the detailed privacy analysis to prove that our algorithm satisfies the differential privacy and proposes a modified moments accountant (MMA) method to get the tighter bound of privacy loss compared with the other popular privacy accounting method.

- We conduct extensive experiments on the classical MNIST, CIFAR-10, and Fashion-MNIST datasets and the results demonstrate that our optimization algorithms can obtain the better model performance over the state-of-the-art algorithms while achieving the equivalent privacy preservation.

The remainder of this article is organized as follows: The related work is presented in Section 2. The background knowledge is introduced in Section 3. A novel perturbed iterative gradient descent optimization algorithm for achieving differentially private deep learning is proposed in Section 4 , where the privacy accounting analysis is also discussed in detail. Subsequently, experimental results are shown in Section 5 to validate the feasibility of our algorithms. We conclude our study in Section 6. 


\section{RELATED WORK}

There have been some studies $[7,11,12,34,35]$ showing that privacy concerns exist in machine learning. For example, Ding et al. [7] exhibited the potential privacy risk in the intermediate layer features of the neural network and they presented two adversarial objectives for different situations, i.e., adopting specific privacy attack as adversarial objective and applying reconstruction attack as adversarial objective. Song et al. [35] illustrated that malicious machine learning algorithms could generate models satisfying the expected quality criteria for accuracy and generality while exposing much information regarding the training datasets, even though the attacker can only access the model in a white-box manner. Therefore, it is necessary for machine or deep learning model to protect privacy. The common privacy-preserving techniques include secure multiparty computation [23], homomorphic encryption [13], and data anonymization [22]. When it comes to deep learning, there have been some recent works [2, 3, 25, 28, 31, 39] utilizing the above techniques to preserve privacy. But these methods have some intrinsic drawbacks. Specifically, secure multiparty computation and homomorphic encryption sometimes require so much computation that the computation burden is unacceptable. The data anonymization model cannot resist homogenous attacks and background knowledge attacks.

To better cope with these privacy concerns, many researchers choose differential privacy as a privacy protection method, since it builds on the solid mathematical foundation and provides a quantitative evaluation of privacy loss. Song et al. [36] presented differentially private versions of stochastic gradient descent to minimize convex loss function in machine learning, but generally, the objective function in deep learning is non-convex. Therefore, some works focused on how to apply differential privacy to deep learning. Shokri et al. [33] proposed a framework called differentially private distributed deep learning framework, where they first used differential privacy for preserving privacy in deep learning, but the privacy loss was too high. Abadi et al. [1] put forward a new algorithm that was based on the differentially private SGD algorithm of Reference [36]. In their work, the authors achieved a privacy guarantee of the training phase by adding noise to the scaled gradients in the SGD process and they developed the moments accountant to obtain the tighter privacy budget.

Recently, there have been some further works on differentially private deep learning $[14,16$, 20, 21, 29, 30, 37, 41, 43, 44]. Most of them are the variants of the private SGD algorithm that is based on the gradient perturbation. Some studies paid attention to the noise addition strategy. For example, Yu et al. [44] investigated differentially private deep learning for model publishing and developed a dynamic privacy budget allocation method that utilized some different techniques to adjust the noise scale during the training process. Xiang et al. [41] presented an optimized additive noise mechanism, where they designedly added more noise to the parameters having less influence on the output to minimize the accuracy loss while meeting the privacy restrictions. Following the work of Reference [41], Xu et al. [43] proposed an adaptive and fast convergent differentially private algorithm that adjusted the learning rate in an adaptive manner and added the sensitivity-dependent noise to obtain the more proper privacy protection. Some other work such as References [14,30] paid attention to the relevance of different features to the model output and presented the adaptive noise addition mechanism based on the relevance analysis. Besides, some researchers undertook their work on privacy loss accounting. For example, Yu et al. [44] took advantage of concentrated differential privacy to obtain a tighter evaluation on privacy loss, because the training of neural networks often has numerous iterations. Lee et al. [20] focused on the modification of clipping and noise to achieve a dynamic allocation of the privacy budget between iterations and used concentrated differential privacy to account the privacy loss. 
As mentioned above, many existing works achieved differentially private deep learning by perturbing the gradient in the SGD algorithm, but the SGD algorithm has some of its own drawbacks such as difficulty in escaping from saddle points. There are few studies considering the other gradient descent optimization algorithms. To our best knowledge, only References [14,19] mentioned the differentially private gradient descent optimization algorithm but they only simply presented the private algorithm that just conducted the single one optimization for the perturbed gradient in each training step and the privacy accounting analysis was not considered in their work. Compared with their works, we integrate the gradient descent optimization algorithm as an iterative component into the differentially private deep learning framework, and it is beneficial for increasing the training speeding and adding the noise adaptively. More importantly, we give the detailed privacy analysis to prove that our algorithm satisfies differential privacy and make some improvements over the latest moments accountant to provide a better privacy accounting method for our differentially private gradient descent optimization algorithm.

\section{BACKGROUND}

In this section, first, we give some definitions and properties about differential privacy. Then, we overview the basic knowledge of deep learning and finally, we provide some description of gradient descent optimization algorithms.

\subsection{Differential Privacy}

Definition 1 (Differential Privacy [9]). A randomized algorithm $\mathcal{A}: \mathcal{X} \rightarrow \mathcal{Y}$, where $\mathcal{X}$ represents the domain and $\mathcal{Y}$ represents the range, achieves $(\varepsilon, \delta)$-differential privacy if for each pair of adjacent datasets $X, X^{\prime} \in \mathcal{X}$ and for every output subset $Y \subseteq \mathcal{Y}$,

$$
\operatorname{Pr}[\mathcal{A}(X) \in Y] \leq e^{\varepsilon} \operatorname{Pr}\left[\mathcal{A}\left(X^{\prime}\right) \in Y\right]+\delta .
$$

In addition to $(\varepsilon, \delta)$-differential privacy, there exists a special case known as $\varepsilon$-differential privacy when $\delta=0$. According to the definition, it can be noted that $\varepsilon$-differential privacy guarantees that the outputs of algorithm $\mathcal{A}$ on any pair of neighboring datasets are nearly equally likely to be observed. However, for $(\varepsilon, \delta)$-differential privacy, it permits to dissatisfy $\varepsilon$-differential privacy with a low probability $\delta$. Therefore, $\varepsilon$-differential privacy is often known as pure differential privacy, while $(\varepsilon, \delta)$-differential privacy is known as approximate differential privacy.

Differential privacy is widely used in privacy protection not only because it provides strict mathematical theory guarantee, but also because it gives a specific privacy protection level that refers to the privacy budget $\varepsilon$. It can be seen that the smaller $\varepsilon$ is, the better privacy guarantee is. In addition to the above reasons, another important reason is that differential privacy is equipped with exquisite composition property. In general, for realizing a complex privacy preserving algorithm, it is necessary to compose differentially private components multiple times. First, each differentially private component is assigned a reasonable privacy budget, and then by utilizing composability, the overall privacy protection level of an algorithm is within the given privacy budget.

For the class of $\left(\varepsilon_{i}, \delta_{i}\right)$-differentially private algorithm, the basic composition theorem [8] states that the composition satisfies $\left(\sum_{i=1}^{k} \varepsilon_{i}, \sum_{i=1}^{k} \delta_{i}\right)$-differential privacy under $k$-fold adaptive composition. Moreover, for each $\varepsilon>0, \delta$, and $\delta^{\prime} \in(0,1]$, let $\varepsilon_{i}=\varepsilon, \delta_{i}=\delta$ and then the strong composition theorem [10] represents that the composition satisfies $\left(k \varepsilon\left(e^{\varepsilon}-1\right)+\varepsilon \sqrt{2 k \log \left(1 / \delta^{\prime}\right)}\right.$, $\left.k \delta+\delta^{\prime}\right)$-differential privacy under $k$-fold adaptive composition.

Definition 2 (Sensitivity [40]). The sensitivity of a query $f: \mathcal{X} \rightarrow \mathcal{Y}$ is

$$
S_{f}=\max _{X, X^{\prime}}\left\|f(X)-f\left(X^{\prime}\right)\right\|,
$$


where $X, X^{\prime}$ are any pair adjacent datasets that differ at most one entry, \|\| represents $\ell_{1}$ or $\ell_{2}$ norm.

A general method for realizing differential privacy is by adding some noise that is calibrated to sensitivity $S_{f}$ to the output. The sensitivity reflects the maximal output change when one single database entry changes and determines that for a specific query, how much noise is needed to achieve differential privacy. In this article, we choose the Gaussian mechanism, which is defined as

$$
\mathcal{G}(X)=f(X)+\mathcal{N}\left(0, S_{f}^{2} \sigma^{2} \mathbf{I}\right),
$$

where $S_{f}$ is chosen as $\ell_{2}$-norm sensitivity and $\mathcal{N}\left(0, S_{f}^{2} \sigma^{2} \mathbf{I}\right)$ denotes the zero-mean Gaussian distribution with variance $S_{f}^{2} \sigma^{2}$. From Reference [9], it can be proved that when $\varepsilon \in(0,1)$, $\sigma^{2} \geq \frac{2 \ln \left(\frac{1.25}{\delta}\right) S_{f}^{2}}{\varepsilon^{2}}$, the Gaussian mechanism satisfies $(\varepsilon, \delta)$-differential privacy.

For the differentially private algorithms, a crucial task is to compute the overall privacy cost during the whole running process. Therefore, it is necessary to define the privacy loss.

Definition 3 (Privacy Loss [9]). For a randomized mechanism $\mathcal{A}: \mathcal{X} \rightarrow \mathcal{Y}$, adjacent databases $X, X^{\prime} \in \mathcal{X}$, auxiliary input aux and an outcome $o \in \mathcal{Y}$, the privacy loss at $o$ is defined as

$$
c\left(o ; \mathcal{A}, \text { aux, } X, X^{\prime}\right) \triangleq \log \frac{\operatorname{Pr}[\mathcal{A}(\operatorname{aux}, X)=o]}{\operatorname{Pr}\left[\mathcal{A}\left(\operatorname{aux}, X^{\prime}\right)=o\right]} .
$$

According to the above definition, a mechanism $\mathcal{A}$ is $(\varepsilon, \delta)$-differential privacy if there is a certain bound on $\mathcal{A}$ 's privacy loss. But as a random variable, privacy loss has a long tail distribution that results in the loose tail bound. For solving this problem, Abadi et al. [1] made use of the moment of the privacy loss random variable to get a shaper tail bound. Moreover, they came up with a technique called moments accountant to compute the accumulated privacy loss under composition. The following are some definitions and properties related to the moments accountant.

Definition 4 ([1]). For a randomized mechanism $\mathcal{A}$, the cumulant generating function (or the $\log$ of the moment generating function) of the privacy loss random variable is defined as

$$
\begin{aligned}
\mathcal{K}_{\mathcal{A}}\left(\lambda ; \operatorname{aux}, X, X^{\prime}\right) & \triangleq \log \mathbb{E}_{o \sim \mathcal{A}(\operatorname{aux}, X)}\left[e^{\lambda c\left(o ; \mathcal{A}, \text { aux }, X, X^{\prime}\right)}\right] \\
& =\log \mathbb{E}_{o \sim \mathcal{A}(\operatorname{aux}, X)}\left[\left(\frac{\operatorname{Pr}[\mathcal{A}(\operatorname{aux}, X)=o]}{\operatorname{Pr}\left[\mathcal{A}\left(\operatorname{aux}, X^{\prime}\right)=o\right]}\right)^{\lambda}\right],
\end{aligned}
$$

where $\lambda$ is the order of the moment.

Property 1 (Composability). Assume that a mechanism $\mathcal{A}$ is made up of a class of adaptive mechanisms $\mathcal{A}_{1}, \ldots, \mathcal{A}_{k}$ where $\mathcal{A}_{i}: \prod_{j=1}^{i-1} \mathcal{Y}_{j} \times \mathcal{X} \rightarrow \mathcal{Y}_{i}$. Then, for every output sequence $o_{1}, \ldots, o_{k-1}$ and each $\lambda$

$$
\mathcal{K}_{\mathcal{A}}\left(\lambda ; X, X^{\prime}\right)=\sum_{i=1}^{k} \mathcal{K}_{\mathcal{A}_{i}}\left(\lambda ; o_{1}, \ldots, o_{i-1}, X, X^{\prime}\right) .
$$

To give the privacy guarantee of a mechanism, it is necessary to bound every possible $\mathcal{K}_{\mathcal{A}}\left(\lambda ;\right.$ aux $\left., X, X^{\prime}\right)$ and define

$$
\mathcal{K}_{\mathcal{A}}(\lambda)=\max _{\text {aux }, X, X^{\prime}} \mathcal{K}_{\mathcal{A}}\left(\lambda ; \text { aux }, X, X^{\prime}\right)
$$

where the maximum is taken over every possible aux and every pair of adjacent datasets $X, X^{\prime}$.

Property 2 (TAIl Bound). For any $\varepsilon>0$, the mechanism $\mathcal{A}$ is $(\varepsilon, \delta)$-differentially private for

$$
\delta=\min _{\lambda} e^{\mathcal{K}_{\mathcal{H}}(\lambda)-\lambda \varepsilon} .
$$




\subsection{Deep Learning}

Deep learning makes use of the nonlinear transformation from original inputs to expected outputs for achieving the nonlinear modelling of input data. In a typical multi-layer neural network, the $m$ th layer of the network is parameterized by a weighted matrix $W^{m}$ as well as a bias vector $b^{m}$. The output in layer $m+1$ denoted by $y^{m+1}$ satisfies $y^{m+1}=f\left(W^{m} y^{m}+b^{m}\right)$, where $f$ is an activation function and the common examples of it are sigmoid, tanh, and rectified linear unit (ReLU).

The goal of training a neural network is that for a given training dataset, the final parameters $\omega=\left\{W^{m}, b^{m} \mid 1 \leq m \leq n\right\}$ can minimize a loss function $\mathcal{L}$ that is used to describe the penalty for mismatching the training data. The loss function of a deep neural network is typically non-convex and hard to minimize. The widely used methods for obtaining the minimization are gradient descent algorithm and its variants. The general algorithm process is described as follows: First, gradient descent begins with a random point. Subsequently, at every step, it computes the gradient of the nonlinear loss function and updates the parameters to make the gradient decrease. Finally, the process goes on until the algorithm converges to a local optimum.

In practice, the minimization is usually achieved by the mini-batch stochastic gradient descent (SGD) algorithm, which is especially suitable for the large dataset. In this algorithm, at each step, a quite small batch (mini-batch) $B$ of examples is randomly sampled from the training dataset and the gradient $\nabla_{\omega} L(\omega)$ is estimated by $g_{B}=1 /|B| \sum_{x \in B} \nabla_{\omega} L\left(\omega_{t}, x\right)$. Then parameters $\omega$ are updated by

$$
\omega_{t+1}=\omega_{t}-\alpha g_{B},
$$

where $\alpha$ is the learning rate. One full iteration over all training examples is called an epoch.

\subsection{Gradient Descent Optimization Algorithms}

SGD algorithm does not always reach the global minimum in reasonable time, so in this article, we introduce some gradient descent optimization (GDO) algorithms, such as Adam, RMSprop, and Adagrad. However, in this subsection, we only give the specific description of Adam, since the basic ideas of the other two algorithms are similar to Adam.

Adam [18] is an adaptive learning rate optimization algorithm that simultaneously stores exponential moving average of the gradient $f_{t}$ and that of the squared gradient $s_{t}$ :

$$
\begin{aligned}
& f_{t}=\beta_{1} f_{t-1}+\left(1-\beta_{1}\right) g_{t} \\
& s_{t}=\beta_{2} s_{t-1}+\left(1-\beta_{2}\right) g_{t}^{2} .
\end{aligned}
$$

Besides, $f_{t}$ and $s_{t}$ are estimates of the first moment (the mean) and the second moment (the uncentered variance) of the gradients, respectively. Because $f_{t}$ and $s_{t}$ are initialized as vectors of 0 's, they are biased to zero when they are during the initial time steps or the decay rates are small. One solution is to counteract this initialization bias by bias-corrected estimates $\hat{f}_{t}$ and $\hat{s}_{t}$,

$$
\hat{f}_{t}=\frac{f_{t}}{1-\beta_{1}^{t}}, \quad \hat{s}_{t}=\frac{s_{t}}{1-\beta_{2}^{t}} .
$$

Then $\hat{f}_{t}$ and $\hat{s}_{t}$ are used to update the parameters, which yields the Adam update rule:

$$
\omega_{t+1}=\omega_{t}-\alpha \frac{\hat{f}_{t}}{\sqrt{\hat{s}_{t}}+a} .
$$


Table 1. Adaptive Iterative GDO

\begin{tabular}{|c|c|c|c|}
\hline & Adagrad & RMSprop & Adam \\
\hline$f_{t}$ & $g_{t}$ & $g_{t}$ & $\frac{\beta_{1} f_{t-1}+\left(1-\beta_{1}\right) g_{t}}{1-\beta_{1}^{t}}$ \\
\hline$s_{t}$ & $s_{t-1}+g_{t}^{2}$ & $\beta_{2} s_{t-1}+\left(1-\beta_{2}\right) g_{t}^{2}$ & $\frac{\beta_{2} s_{t-1}+\left(1-\beta_{2}\right) g_{t}^{2}}{1-\beta_{2}^{t}}$ \\
\hline
\end{tabular}

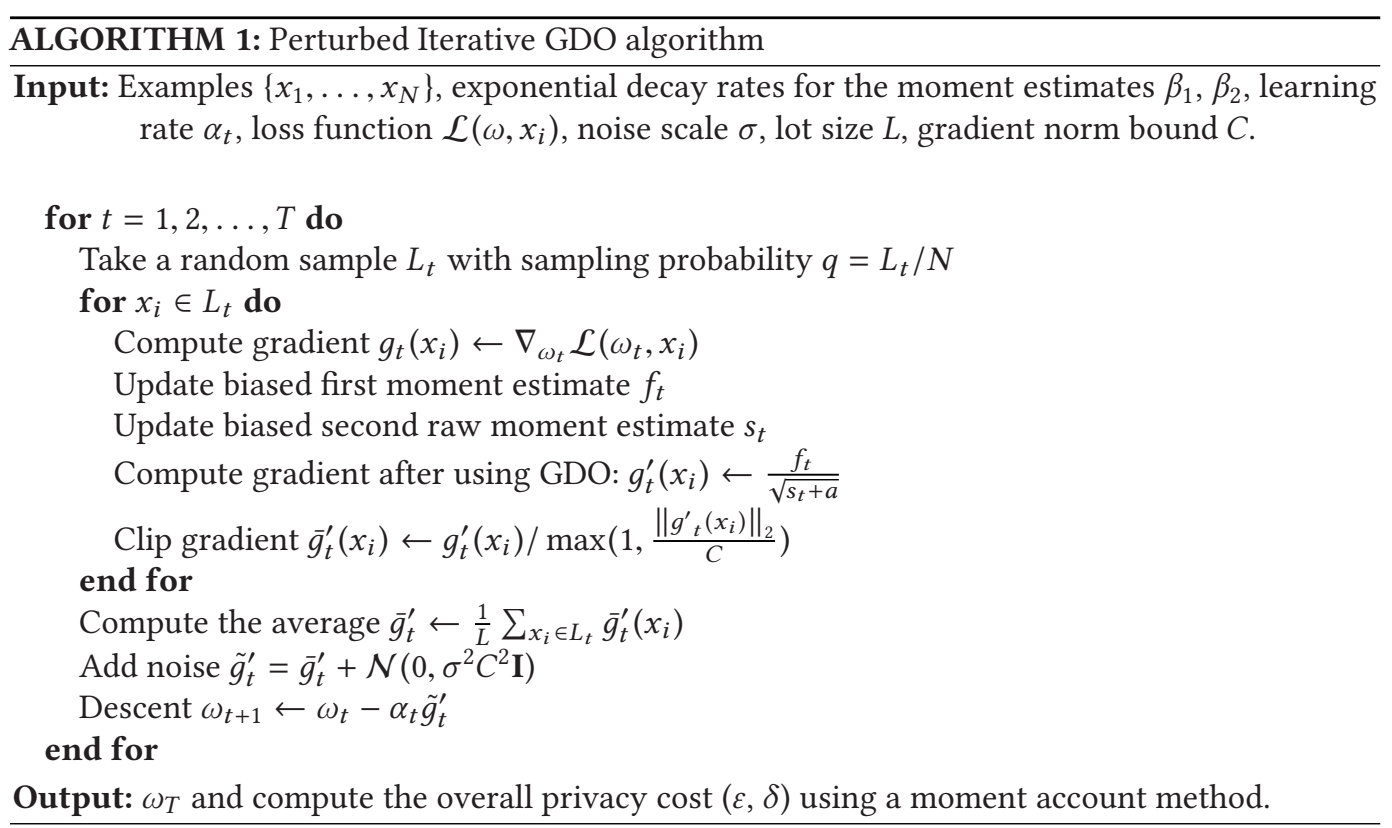

\section{OUR APPROACH}

\subsection{Perturbed Iterative GDO Algorithm (PIGDO)}

In this section, we present the perturbed iterative GDO algorithm to achieve differential privacy. Our PIGDO framework is suitable for three kinds of adaptive GDO algorithms, i.e., Adam, Adagrad, and RMSprop. The detailed algorithm design parameters are provided in Table 1. Algorithm 1 outlines our PIGDO algorithm.

In particular, in this algorithm, there is a training parameter lot size $L$ that is specific to differentially private deep learning. The introduction of the lot is to add noise and it is different from the concept of batch, which is for computing. More specifically, we execute the computing task in batches and then aggregate some batches into a lot to add noise. In each iteration of Algorithm 1 , we randomly select a lot of samples from examples and compute the gradient $g_{t}^{\prime}\left(x_{i}\right)$ of the loss function on these samples by using GDO algorithm. Next, considering that gradient may become very big during the gradient descent process, we cannot get the useful sensitivity of the gradient according to the definition of sensitivity. It brings troubles to Algorithm 1 for achieving differential privacy. To overcome this difficulty, we utilize the gradient clipping to bound each per-example gradient via clipping the $l_{2}$ norm of gradient $g_{t}^{\prime}\left(x_{i}\right)$ with a threshold $C$. In other words, we replace each gradient $g_{t}^{\prime}\left(x_{i}\right)$ with $g_{t}^{\prime}\left(x_{i}\right) / \max \left(1, \frac{\left\|g_{t}^{\prime}\left(x_{i}\right)\right\|_{2}}{C}\right)$ to scale $g_{t}^{\prime}\left(x_{i}\right)$ down to norm $C$ when $\left\|g_{t}^{\prime}\left(x_{i}\right)\right\|_{2}>C$. After that, we compute the average of these clipping gradients and add random noise $\mathcal{N}\left(0, \sigma^{2} C^{2} \mathbf{I}\right)$ to $\bar{g}_{t}^{\prime}$ to perturb the average. Finally, we update the model parameter with noisy gradient $\tilde{g}_{t}^{\prime}$ at 


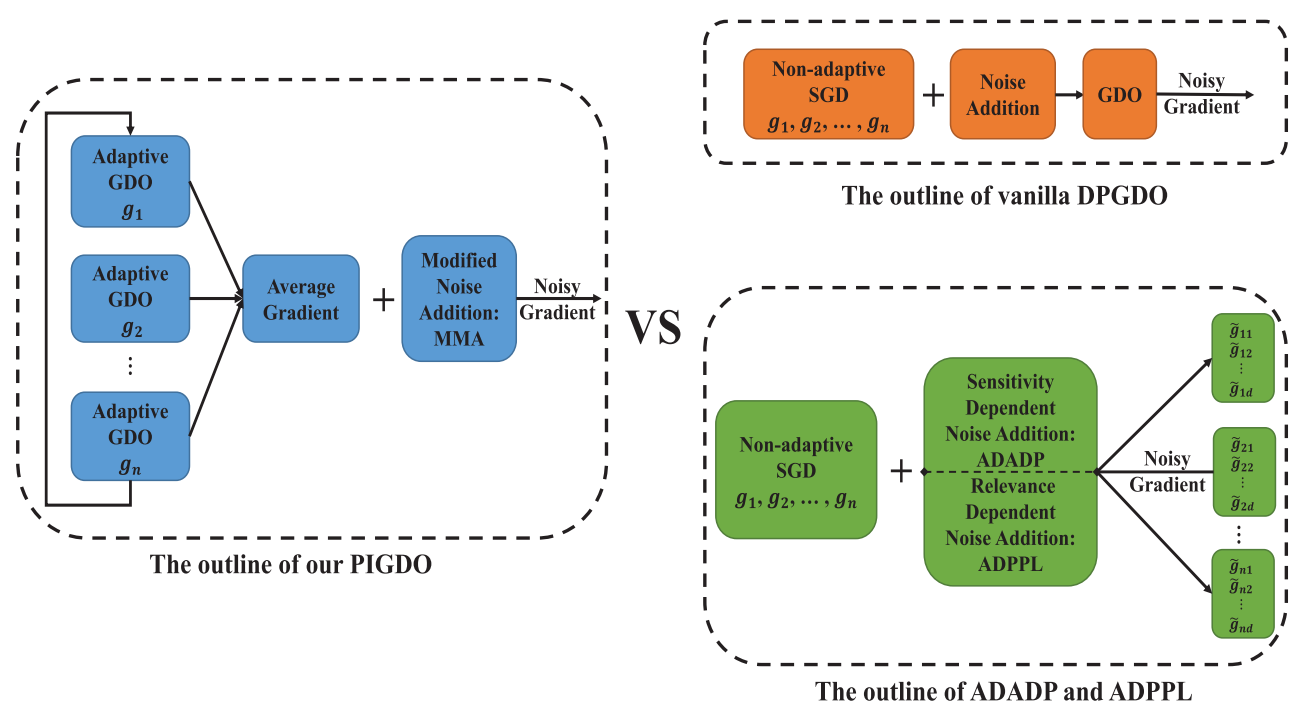

Fig. 2. Comparing our PIGDO algorithm with the state-of-the-art gradient perturbation-based algorithms: DPGDO [19], ADADP [43], ADPPL [14].

each iteration step. As every iteration satisfies differential privacy, the final model parameters also satisfy differential privacy based on the composition property of differential privacy.

Remark 1. Several recent works also aim to optimize the differentially private deep learning algorithm by injecting the proper noise into the gradient. They mainly focus on the gradient perturbation based on the sensitivity analysis of each gradient [41, 43] or the relevance analysis of each feature to the model output $[14,30]$, and these methods have some defects. Specifically, the necessary condition in Reference [43] is difficult to protect privacy under the high-dimensional deep learning structure. In Reference [41], solving a large-scale optimization problem per step causes costly computation. In References [14, 30], the calculation for the relevance of each feature in different neural network layers causes inefficient computation. Although our method adds the same amount of noise to all gradients, we can still get the equivalent performance compared with these latest methods. This is because the integration of the adaptive GDO into every iteration can get better overall accuracy via fewer iterations but faster convergence. Besides, we only need to inject less noise into the algorithm for protecting privacy.

Remark 2. A few works [14, 19] also discussed the DP gradient descent optimization method. There exists a main difference between our work with theirs. Our work integrates the gradient descent optimization method as an iterative process into each differentially private noise addition step. However, their works just conducted the single one optimization for the perturbed gradient in one iteration step. Compared with their work, the distinct advantage brought by this difference is that we can adaptively add the noise through the GDO method and get the better algorithm performance. Although in our algorithm, the iterative process for the gradient descent optimization brings more noise injections and leads to the more challenging privacy budget analysis, we conduct the detailed privacy analysis in the next subsection to prove that our PIGDO method can satisfy the DP guarantee. To explain the difference between our algorithm and the above algorithms more clearly, we present Figure 2. 


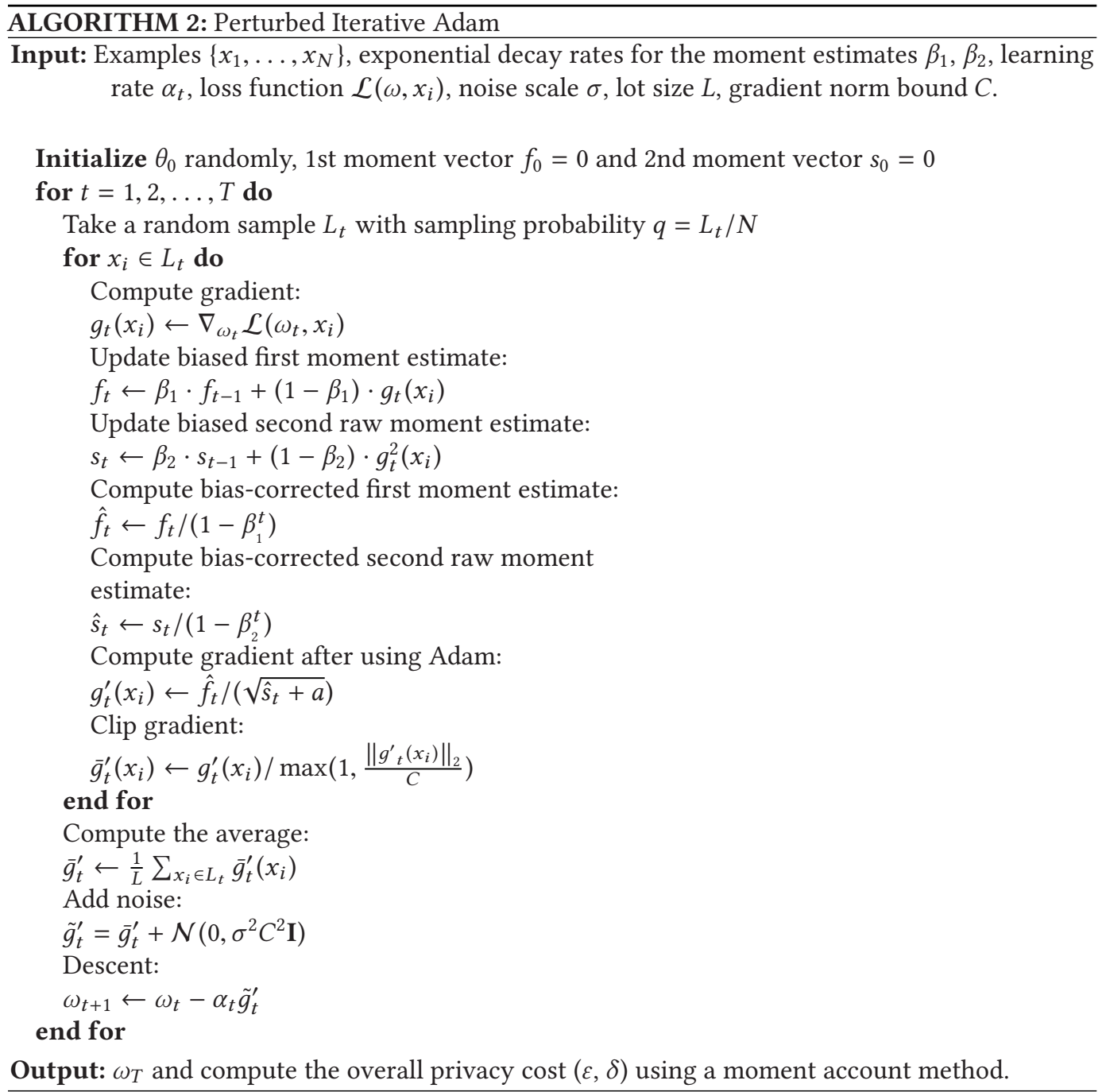

\subsection{Privacy Analysis}

Except for outputting the model, it is also necessary for us to study the privacy loss of PIGDO algorithm. In the following discussion, we only give the detailed algorithm for PIAdam shown in Algorithm 2 and the overall algorithm procedures of PIAdagrad and PIRMSprop are similar and therefore omitted on account of space limitations. In this algorithm, for the training of a differentially private deep learning model, it typically requires many iteration steps, which will eventually result in a large overall privacy loss. Hence, we need to propose a suitable privacy loss accounting method to track privacy loss. There have been some approaches to account for the privacy loss, and the state-of-the-art technique is the moments accountant method presented by Reference [1], which achieved a much tighter estimate of privacy loss than the strong composition theorem. In the following, we propose a modified moments accountant method (MMA) that improves their moments accountant method and present Theorem 3 to prove that PIAdam satisfies differential privacy and provides a tighter overall privacy loss. 
Theorem 3. Given the sampling probability $q=L / N$ and the number of steps $T$, when noise scale $\sigma \geq 1$, sample probability $q<1 / \sigma$ and the order of moment $\lambda \leq \frac{1}{10 q^{2}}$, for any $\varepsilon>0.002 T / \lambda$, if we choose $\sigma \geqslant \frac{2 q \log \frac{1}{\delta}}{\varepsilon \sqrt{\delta^{-\frac{1}{T}}-1}}$, then Algorithm 2 is $(\varepsilon, \delta)$-differential privacy for any $\delta>0$.

Proof. For convenience, we use $\mathcal{G}_{s}$ to represent $\tilde{g}_{t}^{\prime}=\bar{g}_{t}^{\prime}+\mathcal{N}\left(0, \sigma^{2} C^{2} \mathbf{I}\right)$ in Algorithm 2, i.e.,

$$
\mathcal{G}_{s}=\frac{1}{L} \sum_{x_{i} \in L_{t}} \bar{g}_{t}^{\prime}\left(x_{i}\right)+\mathcal{N}\left(0, \sigma^{2} C^{2} \mathbf{I}\right) .
$$

In fact, we can regard $\mathcal{G}_{s}$ as a differential privacy mechanism under random sampling with replacement. It means that differential privacy mechanism $\mathcal{G}_{s}$ runs on a random subsample of dataset $X$ where each example in $L_{t}$ is independently sampled with probability $q$. Next, we introduce the privacy loss to analyze the condition guaranteeing the differential privacy.

- Formulate the privacy loss. First, we consider the uncertainty brought by random sampling. For convenience of analysis, we fix dataset $X$ and study a neighboring dataset $X^{\prime}=X \cup x_{e}$. We use $S(*)$ to represent the sampling process over the dataset. It can be noted that all the $L_{t}$ sampled from $X^{\prime}=X \cup x_{e}$ with probability $q$ can be divided into two cases: (1) $x_{e}$ is not sampled and (2) $x_{e}$ is sampled. To illustrate the sampling process more clearly, we denote $T$ as any subsample that does not include $x_{e}$ and express $T^{\prime}$ as $T \cup x_{e}$. Because $x_{e}$ is randomly sampled with probability $q$, for the case (1), we have

$$
\begin{aligned}
& \operatorname{Pr}\left[S\left(X^{\prime}\right)=L_{t}\right] \\
= & (1-q) \operatorname{Pr}\left[S\left(X^{\prime}\right)=T \mid x_{e} \text { not sampled in } L_{t}\right] \\
= & (1-q) \operatorname{Pr}[S(X)=T],
\end{aligned}
$$

and for the case (2), we have

$$
\operatorname{Pr}\left[S\left(X^{\prime}\right)=L_{t}\right]=q \operatorname{Pr}\left[S\left(X^{\prime}\right)=T^{\prime} \mid x_{e} \text { sampled in } L_{t}\right] .
$$

Then, for the mechanism $\mathcal{G}_{s}$ on dataset $X$ and $X^{\prime}$, we have

$$
\mathcal{G}_{s}(X)=\operatorname{Pr}[S(X)=T] \cdot \mathcal{G}_{s}(T),
$$

and

$$
\mathcal{G}_{s}\left(X^{\prime}\right)=(1-q) \operatorname{Pr}[S(X)=T] \cdot \mathcal{G}_{s}(T)+q \operatorname{Pr}\left[S\left(X^{\prime}\right)=T^{\prime}\right] \cdot \mathcal{G}_{s}\left(T^{\prime}\right) .
$$

Next, we begin to focus on the privacy loss of mechanism $\mathcal{G}_{s}$ and according to Definition 3, we need to consider $\frac{\operatorname{Pr}\left[\mathcal{G}_{s}(X)\right]}{\operatorname{Pr}\left[\mathcal{G}_{s}\left(X^{\prime}\right)\right]}$. Because from the perspective of probability, $\mathcal{G}_{s}=\frac{1}{L} \sum_{x_{i} \in L_{t}} \bar{g}_{t}^{\prime}\left(x_{i}\right)+$ $\mathcal{N}\left(0, \sigma^{2} C^{2} \mathbf{I}\right)$ is equally likely with $\sum_{x_{i} \in L_{t}} \bar{g}_{t}^{\prime}\left(x_{i}\right)+\mathcal{N}\left(0, \sigma^{2} C^{2} \mathbf{I}\right)$, we can reconsider the subsampled mechanism as

$$
\mathcal{G}=\sum_{x_{i} \in L_{t}} \bar{g}_{t}^{\prime}\left(x_{i}\right)+\mathcal{N}\left(0, \sigma^{2} C^{2} \mathbf{I}\right)
$$

Without loss of generality, we suppose $\|\mathcal{G}(\cdot)\|_{2} \leq 1, \sum_{x_{i} \in L_{t}} \bar{g}_{t}^{\prime}\left(x_{i}\right)=0$ when $L_{t}$ is sampled from $X$ and $\bar{g}_{t}^{\prime}\left(x_{e}\right)=\mathbf{e}_{1}$ (unit vector), then in (1) and (2), $\mathcal{G}_{s}(T) \sim N\left(0, \sigma^{2}\right)$ and $\mathcal{G}_{s}\left(T^{\prime}\right) \sim N\left(1, \sigma^{2}\right)$. Moreover, $\operatorname{Pr}[S(X)=T]=\operatorname{Pr}\left[S\left(X^{\prime}\right)=T^{\prime} \mid x_{e} \in T^{\prime}\right]$ due to $T^{\prime}=T \cup x_{e}$. Substituting the above equations into (1) and (2), we have

$$
\frac{\operatorname{Pr}\left[\mathcal{G}_{s}(X)\right]}{\operatorname{Pr}\left[\mathcal{G}_{s}\left(X^{\prime}\right)\right]}=\frac{\mathcal{G}(T)}{(1-q) \mathcal{G}(T)+q \mathcal{G}\left(T^{\prime}\right)} .
$$

For simplicity, we denote $\mathcal{G}(T) \sim N\left(0, \sigma^{2}\right)$ as $u_{0}, \mathcal{G}\left(T^{\prime}\right) \sim N\left(1, \sigma^{2}\right)$ as $u_{1}$ and $(1-q) u_{0}+q u_{1} \triangleq u$. Therefore, privacy loss $c \sim \frac{u_{0}}{u}$. 
- Analyze the upper bound of privacy loss. As a random variable, privacy loss has a long tail distribution that results in the loose tail bound. For solving this problem, we utilize the moment of the privacy loss random variable to get a shaper tail bound. According to Property 2, it can be seen that when we get a bound of the cumulant generating function $\mathcal{K}_{\mathcal{G}}(\lambda)$, Algorithm 2 will satisfy certain $(\varepsilon, \delta)$-differential privacy. Therefore, from Definition 4 , we need to prove $\mathbb{E}_{z \sim u_{0}}\left[\left(\frac{u_{0}(z)}{u(z)}\right)^{\lambda}\right] \leqslant$ $\alpha$, where $\alpha$ denotes a specific upper bound.

Now consider

Using binomial expansion, we have

$$
\mathbb{E}_{z \sim u_{0}}\left[\left(\frac{u_{0}(z)}{u(z)}\right)^{\lambda}\right]=\mathbb{E}_{z \sim u}\left[\left(\frac{u_{0}(z)}{u(z)}\right)^{\lambda+1}\right]
$$

$$
\begin{aligned}
\mathbb{E}_{z \sim u}\left[\left(\frac{u_{0}(z)}{u(z)}\right)^{\lambda+1}\right] & =\mathbb{E}_{z \sim u}\left[\left(1+\frac{u_{0}(z)-u(z)}{u(z)}\right)^{\lambda+1}\right] \\
& =\sum_{t=0}^{\lambda+1} C_{\lambda+1}^{t} \mathbb{E}_{z \sim u}\left[\left(\frac{u_{0}(z)-u(z)}{u(z)}\right)^{t}\right] .
\end{aligned}
$$

The first term $(t=0)$ in (3) is 1 and the multiplier of second term $(t=1)$ in (3) is

$$
\begin{aligned}
\mathbb{E}_{z \sim u}\left[\frac{u_{0}(z)-u(z)}{u(z)}\right] & =\int_{-\infty}^{+\infty} u(z) \cdot \frac{u_{0}(z)-u(z)}{u(z)} d z \\
& =\int_{-\infty}^{+\infty} u_{0}(z) d z-\int_{-\infty}^{+\infty} u(z) d z=1-1=0 .
\end{aligned}
$$

Then the second term in (3) is 0 and the third term $(t=2)$ in (3) is

$$
C_{\lambda+1}^{2} \mathbb{E}_{z \sim u}\left[\left(\frac{u_{0}(z)-u(z)}{u(z)}\right)^{2}\right] .
$$

To give the upper bound of the third term, we note that $(1-q) u_{0}(z)+q u_{1}(z)=u(z)$ and then $u(z) \geqslant(1-q) u_{0}(z)$. Therefore, we can obtain

$$
\begin{aligned}
\mathbb{E}_{z \sim u}\left[\left(\frac{u_{0}(z)-u(z)}{u(z)}\right)^{2}\right] & =q^{2} \mathbb{E}_{z \sim u}\left[\left(\frac{u_{0}(z)-u_{1}(z)}{u(z)}\right)^{2}\right] \\
& =q^{2} \int_{-\infty}^{+\infty} \frac{\left(u_{0}(z)-u_{1}(z)\right)^{2}}{u(z)} d z \\
& \leq \frac{q^{2}}{1-q} \int_{-\infty}^{+\infty} \frac{\left(u_{0}(z)-u_{1}(z)\right)^{2}}{u_{0}(z)} d z \\
& =\frac{q^{2}}{1-q} \mathbb{E}_{z \sim u_{0}}\left[\left(\frac{u_{0}(z)-u_{1}(z)}{u_{0}(z)}\right)^{2}\right] .
\end{aligned}
$$

It is easy to prove that for any $a \in \mathbb{R}$, when $z \sim \mathcal{N}\left(0, \sigma^{2}\right), \mathbb{E}_{z \sim u_{0}} e^{\frac{2 a z}{2 \sigma^{2}}}=e^{\frac{a^{2}}{2 \sigma^{2}}}$. Thus,

$$
\begin{aligned}
\mathbb{E}_{z \sim u_{0}}\left[\left(\frac{u_{0}(z)-u_{1}(z)}{u_{0}(z)}\right)^{2}\right] & =\mathbb{E}_{z \sim u_{0}}\left[\left(1-e^{\frac{2 z-1}{2 \sigma^{2}}}\right)^{2}\right] \\
& =1-2 \mathbb{E}_{z \sim u_{0}}\left[e^{\frac{2 z-1}{2 \sigma^{2}}}\right]+\mathbb{E}_{z \sim u_{0}}\left[e^{\frac{4 z-2}{2 \sigma^{2}}}\right] \\
& =1-2 e^{\frac{1}{2 \sigma^{2}}} \cdot e^{\frac{-1}{2 \sigma^{2}}}+e^{\frac{4}{2 \sigma^{2}}} \cdot e^{\frac{-2}{2 \sigma^{2}}}=e^{\frac{1}{\sigma^{2}}}-1 .
\end{aligned}
$$


Although from the viewpoint of mathematics, the upper bound of $\left(e^{\frac{1}{\sigma^{2}}}-1\right)$ does not exist, Algorithm 2 has a limitation on noise scale $\sigma$. For providing proper privacy protection, the noise should not be too small so we give a bound of $\sigma$ as $\sigma \geq 1$ in this article. Then, we can get

$$
e^{\frac{1}{\sigma^{2}}}-1 \leqslant(e-1) \frac{1}{\sigma^{2}}
$$

Substituting (7) into (6), (6) into (5), and (5) into (4), it can be seen that the third term in (3) satisfies

$$
\begin{aligned}
& C_{1+\lambda}^{2} \mathbb{E}_{z \sim u}\left[\left(\frac{u_{0}(z)-u(z)}{u(z)}\right)^{2}\right] \\
\leqslant & \frac{(1+\lambda) \lambda}{2} \cdot \frac{q^{2}}{1-q} \cdot \frac{e-1}{\sigma^{2}}=\frac{e-1}{2} \cdot \frac{\lambda(\lambda+1) q^{2}}{(1-q) \sigma^{2}} \leqslant \frac{q^{2} \lambda^{2}}{\sigma^{2}} .
\end{aligned}
$$

Now, we make use of an important conclusion that when $z \sim \mathcal{N}\left(0, \sigma^{2}\right), \mathbb{E}_{z \sim u_{0}}\left(|z|^{t}\right)=\sigma^{t} \cdot(t-1) !$ !.

Subsequently, we begin to discuss the remaining terms $(t \geq 3)$ in $\mathbb{E}_{z \sim u}\left[\left(\frac{u_{0}(z)-u(z)}{u(z)}\right)^{t}\right]$. To bound the remaining terms, we first note that

OBSERVATION 1. For $\left|\frac{u_{0}(z)-u_{1}(z)}{u_{0}(z)}\right|=\left|1-e^{\frac{2 z-1}{2 \sigma^{2}}}\right|$, only when $z \leq \frac{1}{2}$, it has upper bound $\frac{1-z}{\sigma^{2}}$;

OBSERvation 2. Observation 2: For $\left|\frac{u_{0}(z)-u_{1}(z)}{u_{1}(z)}\right|=\left|e^{\frac{1-2 z}{2 \sigma^{2}}}-1\right|$, only when $z \geq \frac{1}{2}$, it has upper bound $\frac{z}{\sigma^{2}}$.

Therefore, we bound the remaining terms as the following three parts:

$$
\begin{aligned}
& \mathbb{E}_{z \sim u}\left[\left(\frac{u_{0}(z)-u(z)}{u(z)}\right)^{t}\right] \leqslant \int_{-\infty}^{+\infty} u(z)\left|\frac{u_{0}(z)-u(z)}{u(z)}\right|^{t} d z \\
& \leqslant \int_{-\infty}^{0} u(z)\left|\frac{u_{0}(z)-u(z)}{u(z)}\right|^{t} d z+\int_{0}^{1} u(z)\left|\frac{u_{0}(z)-u(z)}{u(z)}\right|^{t} d z+\int_{1}^{\infty} u(z)\left|\frac{u_{0}(z)-u(z)}{u(z)}\right|^{t} d z .
\end{aligned}
$$

We consider these parts individually and repeatedly make use of three conclusions: (1) $u_{0}-u=$ $q\left(u_{0}-u_{1}\right),(2) u \geqslant(1-q) u_{0},(3) \mathbb{E}_{u_{0}}\left(|z|^{t}\right) \leqslant \sigma^{t}(t-1) ! !$.

For the first part,

$$
\begin{aligned}
\int_{-\infty}^{0} u\left|\frac{u_{0}-u}{u}\right|^{t} d z< & \frac{q^{t}}{(1-q)^{t-1}} \int_{-\infty}^{0} u_{0}\left|\frac{u_{0}-u_{1}}{u_{0}}\right|^{t} d z \\
& <\frac{q^{t}}{(1-q)^{t-1}} \int_{-\infty}^{0} u_{0}\left|\frac{1-z}{\sigma^{2}}\right|^{t} d z \\
& =\frac{q^{t}}{(1-q)^{t-1} \cdot \sigma^{2 t}} \int_{-\infty}^{0} u_{0}|z-1|^{t} d z,
\end{aligned}
$$

where conclusion (1), (2), and Observation 1 are used. As we can see that $|z-1|^{t} \leqslant 2^{t}\left(|z|^{t}+1\right)$, therefore, (10) satisfies that

$$
\begin{aligned}
(10) & \leqslant \frac{q^{t}}{(1-q)^{t-1} \cdot \sigma^{2 t}} \int_{-\infty}^{0} u_{0}\left(2^{t}\left(|z|^{t}+1\right)\right) d z \\
& =\frac{(2 q)^{t}}{(1-q)^{t-1} \cdot \sigma^{2 t}} \int_{-\infty}^{0}\left(u_{0}|z|^{t}+u_{0}\right) d z \\
& =\frac{(2 q)^{t}}{2(1-q)^{t-1} \cdot \sigma^{2 t}}\left(\sigma^{t}(t-1) ! !+1\right),
\end{aligned}
$$

where conclusion (3) is used. 
Thus, the upper bound of the first part in (9) is

$$
\frac{(2 q)^{t}}{2(1-q)^{t-1} \cdot \sigma^{2 t}}\left(\sigma^{t}(t-1) ! !+1\right) \text {. }
$$

For the second part,

$$
\int_{0}^{1} u\left|\frac{u_{0}-u}{u}\right|^{t} d z \leqslant \frac{q^{t}}{(1-q)^{t}} \int_{0}^{1} u\left|\frac{u_{0}-u_{1}}{u_{0}}\right|^{t}
$$

where conclusion (1) and (2) are used. When $0<z<1$,

$$
\begin{aligned}
\left|\frac{u_{0}-u_{1}}{u_{0}}\right|= & \left|1-e^{\frac{2 z-1}{2 \sigma^{2}}}\right| \leqslant \max \left\{1-e^{\frac{-1}{2 \sigma^{2}}}, e^{\frac{1}{2 \sigma^{2}}}-1\right\} \\
& =e^{\frac{1}{2 \sigma^{2}}}-1 .
\end{aligned}
$$

In this article, $\sigma \geq 1$ then $e^{\frac{1}{2 \sigma^{2}}}-1<\frac{1}{\sigma^{2}}$. Thus, (13) satisfies

$$
(13) \leqslant \frac{q^{t}}{(1-q)^{t}} \cdot \int_{0}^{1} u \frac{1}{\sigma^{2 t}} d t<\frac{q^{t}}{(1-q)^{t} \sigma^{2 t}} .
$$

Therefore, the upper bound of the second part in (9) is

$$
\frac{q^{t}}{(1-q)^{t} \sigma^{2 t}}
$$

For the third part, we have

$$
\begin{aligned}
& \int_{1}^{\infty} u\left|\frac{u_{0}-u}{u}\right|^{t} d z \leqslant \frac{q^{t}}{(1-q)^{t-1}} \int_{1}^{\infty} u_{0}\left|\frac{u_{0}-u_{1}}{u_{0}}\right|^{t} d z \\
& \leqslant \frac{q^{t}}{(1-q)^{t-1}} \int_{1}^{\infty} u_{0}\left|\frac{\frac{z}{\sigma^{2}} \cdot u_{1}}{u_{0}}\right|^{t} d z \\
& =\frac{q^{t}}{(1-q)^{t-1} \cdot \sigma^{2 t}} \int_{1}^{\infty} u_{0}\left(\frac{z u_{1}}{u_{0}}\right)^{t} d z \\
& =\frac{q^{t}}{(1-q)^{t-1} \cdot \sigma^{2 t}} \int_{1}^{\infty} u_{0} \cdot e^{\frac{2 t z-t}{2 \sigma^{2}}} \cdot z^{t} d z,
\end{aligned}
$$

where conclusions (1), (2), and Observation 2 are used. Since

$$
u_{0} \cdot e^{\frac{2 t z-t}{2 \sigma^{2}}}=\frac{e^{-\frac{(z-t)^{2}}{2 \sigma^{2}}}}{\sqrt{2 \pi} \sigma} \cdot e^{\frac{t^{2}-t}{2 \sigma^{2}}}=u_{0}(z-t) \cdot e^{\frac{t^{2}-t}{2 \sigma^{2}}}
$$

then (15) satisfies

$$
\begin{aligned}
(15) & =\frac{q^{t}}{(1-q)^{t-1} \cdot \sigma^{2 t}} \int_{1}^{\infty} u_{0}(z-t) \cdot e^{\frac{t^{2}-t}{2 \sigma^{2}}} \cdot z^{t} d z \\
& =\frac{q^{t} e^{\frac{t^{2}-t}{2 \sigma^{2}}}}{(1-q)^{t-1} \sigma^{2 t}} \int_{1}^{\infty} u_{0}(z-t) \cdot z^{t} d z .
\end{aligned}
$$


We can note that $z^{t} \leqslant 2^{t}\left[(z-t)^{t}+t^{t}\right]$ for $z \geqslant 0$. Therefore, we have

$$
\begin{aligned}
\int_{1}^{\infty} u_{0}(z-t) \cdot z^{t} d z & \leq \int_{1}^{\infty} u_{0}(z-t) \cdot 2^{t}\left[(z-t)^{t}+t^{t}\right] d z \\
& =2^{t}\left[\int_{1}^{\infty} u_{0}(z-t) \cdot(z-t)^{t} d z+\int_{1}^{\infty} u_{0}(z-t) \cdot t^{t} d z\right] \\
& \leqslant 2^{t}\left[\int_{0}^{\infty} u_{0}(z-t) \cdot(z-t)^{t} d z+t^{t} \cdot \frac{1}{2}\right] \\
& =2^{t}\left[\frac{\sigma^{t}(t-1) ! !}{2}+\frac{1}{2} t^{t}\right]
\end{aligned}
$$

where conclusion (3) is used.

Substituting this inequality into (16), then the upper bound of the third part in (9) is

$$
\frac{(2 q)^{t} e^{\frac{t^{2}-t}{2 \sigma^{2}}}\left[\sigma^{t}(t-1) ! !+t^{t}\right]}{2(1-q)^{t-1} \sigma^{2 t}}
$$

Until now, we have given the rough upper bound of all the part composing $\mathbb{E}_{z \sim u}\left[\left(\frac{u_{0}(z)-u(z)}{u(z)}\right)^{t}\right]$ when $t \geq 3$.

- Modify the upper bound of privacy loss. Next, we want to find a dominant term of this upper bound so we can obtain an asymptotic upper bound. First, intuitively, when the lot size is small, a greater amount of noise is accumulated to the model. Therefore, intuitively, $q$ is inversely proportional to $\sigma$, i.e., $q \sigma<1$. Second, for the bounds of these three integral terms (12), (14), and (17), if these bounds for $t=t$ are more than 10 times that for $t=t+1$, we can only keep the dominant term and the latter terms can be omitted.

Taking (14) as an example, if we want to have a dominant term, we need $\frac{\frac{q^{t}}{(1-q)^{t} \sigma^{2 t}} \mid t=t}{\frac{q^{t}}{(1-q)^{t} \sigma^{2 t}} \mid t=t+1}=$ $\frac{(1-q) \sigma^{2}}{q}>10$, then we have $\sigma^{2}>\frac{10 q}{1-q}$. From the former analysis, we have drawn a conclusion that $q<\frac{1}{\sigma}$. Besides, theoretically, the sampling probability $q$ should satisfy $q \in(0,1)$, but practically, $q$ is typically less than 0.1 . Under this condition, $\frac{1}{q^{2}}>\sigma^{2}>\frac{10 q}{1-q}$ always holds. Therefore, when $\sigma<1 / q$, we can find proper $(q, \sigma)$ to make (14) is dominated at $t=3$. Similarly, for (12) and (17), we can also find the proper $(q, \sigma)$ to guarantee that (12) and (17) are dominated at $t=3$, i.e., we can omit the remaining terms for $t>3$. From the above, it can be concluded that when $q<\frac{1}{\sigma}$, for $t \geq 3$, we can find the proper $(q, \sigma)$ to make $\mathbb{E}_{z \sim u}\left[\left(\frac{u_{0}(z)-u(z)}{u(z)}\right)^{t}\right]$ is dominated at $t=3$, which means that all the remaining terms for $t \geq 3$ can be replaced by

$$
\mathbb{E}_{z \sim u}\left[\left(\frac{u_{0}(z)-u(z)}{u(z)}\right)^{3}\right]=C_{\lambda+1}^{3}[(12)+(14)+(17)]_{t=3} .
$$

It is obvious that (12), (14), and (17) for $\mathrm{t}=3$ are all less than $O\left(\frac{q^{3}}{\sigma^{3}}\right)$ and $C_{\lambda+1}^{3}=\frac{(\lambda+1) \lambda(\lambda-1)}{3 \times 2 \times 1}<$ $\lambda^{3}$, then we can obtain $C_{\lambda+1}^{3} \mathbb{E}_{z \sim u}\left[\left(\frac{u_{0}(z)-u(z)}{u(z)}\right)^{3}\right]=O\left(\frac{\lambda^{3} q^{3}}{\sigma^{3}}\right)$. Moreover, when $t=2, C_{1+\lambda}^{2} \mathbb{E}_{z \sim u}$ $\left[\left(\frac{u_{0}(z)-u(z)}{u(z)}\right)^{2}\right]=O\left(\frac{q^{2} \lambda^{2}}{\sigma^{2}}\right)$, which is given in (8). Thus, to give a more specific upper bound, we give the conditions of these parameters $q, \sigma, \lambda$ to make the term at $t=2$ to be the most dominant term. According to $\frac{q^{2} \lambda^{2}}{\sigma^{2}} / \frac{q^{3} \lambda^{3}}{\sigma^{3}}=\frac{\sigma}{q \lambda}$ and $\sigma<\frac{1}{q}$, then $\frac{\sigma}{q \lambda}<\frac{1}{q^{2} \lambda}$. To guarantee the upper bound for $t=2$ is the most dominant, we need $\frac{1}{q^{2} \lambda}>10$, i.e., $\lambda<\frac{1}{10 q^{2}}$. 
As has been stated above, when $\sigma>1, q<1 / \sigma$, and $\lambda<\frac{1}{10 q^{2}}$, we can replace all the remaining terms for $t \geq 2$ by the upper bound of the third term $(t=2)$ in (3), i.e., $\frac{q^{2} \lambda^{2}}{\sigma^{2}}$. Now, we can obtain that for (3), the first term $(t=0)$ is 1 and the second term $(t=1)$ is 0 . Moreover, all the remaining terms for $t \geq 2$ is $\frac{q^{2} \lambda^{2}}{\sigma^{2}}$. Therefore, we have

$$
\mathbb{E}_{z \sim u_{0}}\left[\left(\frac{u_{0}(z)}{u(z)}\right)^{\lambda}\right]=\mathbb{E}_{z \sim u}\left[\left(\frac{u_{0}(z)}{u(z)}\right)^{\lambda+1}\right]=1+\frac{q^{2} \lambda^{2}}{\sigma^{2}} .
$$

Therefore, from Definition 4, in Algorithm 2, the cumulant generating function of the privacy loss at each step is $\mathcal{K}_{\mathcal{G}}(\lambda)=\log \left(1+\frac{q^{2} \lambda^{2}}{\sigma^{2}}\right)$ and then the overall privacy loss is $\mathcal{K}_{\mathcal{G}}(\lambda)=T \log \left(1+\frac{q^{2} \lambda^{2}}{\sigma^{2}}\right)$.

- Present the condition for achieving $(\varepsilon, \delta)$-differential privacy. From Property 1 and Property 2, we have $\delta=\min _{\lambda} e^{\mathcal{K}_{\mathcal{G}}(\lambda)-\lambda \varepsilon}$. It is obvious that $0<\delta<1$ and then we need $\mathcal{K}_{\mathcal{G}}(\lambda)-\lambda \varepsilon<0$, i.e., $\varepsilon>\mathcal{K}_{\mathcal{G}}(\lambda) / \lambda$. Next, we maintain $\mathcal{K}_{\mathcal{G}}(\lambda)$ for different $\lambda$ to get the best $\delta$ for any given $\varepsilon$ and we obtain $\mathcal{K}_{\mathcal{G}}(\lambda)<\lambda \varepsilon / 2$. Then, we have $T \log \left(1+\frac{q^{2} \lambda^{2}}{\sigma^{2}}\right)<\frac{\lambda \varepsilon}{2}$ and from the above analysis, $\frac{\sigma}{q \lambda}>10$, therefore, we can obtain $\varepsilon>0.002 T / \lambda$. Next, we make use of

$$
\left\{\begin{array}{l}
\mathcal{K}_{\mathcal{G}}(\lambda)=T \log \left(1+\frac{q^{2} \lambda^{2}}{\sigma^{2}}\right)<\frac{\lambda \varepsilon}{2} \\
\delta \leqslant e^{\mathcal{K}_{\mathcal{G}}(\lambda)-\lambda \varepsilon}<e^{-\frac{\lambda \varepsilon}{2}} .
\end{array}\right.
$$

Eliminate $\lambda$ and we can obtain that when $\sigma \geqslant \frac{2 q \log \frac{1}{\delta}}{\sqrt{\delta^{-\frac{1}{T}}-1}}$, the above two inequalities hold simultaneously.

From the above proof, we can get when noise scale $\sigma \geq 1$, sample probability $q<1 / \sigma$ and the order of moment $\lambda \leq \frac{1}{10 q^{2}}$, for any $\varepsilon>0.002 T / \lambda$, if we give the number of steps $T$, sample probability $q$ and choose $\sigma \geqslant \frac{2 q \log \frac{1}{\delta}}{\varepsilon \sqrt{\delta^{-\frac{1}{T}}-1}}$, then Algorithm 2 is $(\varepsilon, \delta)$-differential privacy.

Remark 3. It should be noted that in the above proof, we use a generalized differential privacy mechanism to replace the differentially private gradient descent optimization algorithm. Therefore, although we only give the theorem for the PIAdam algorithm, our Theorem 3 is also suitable for the other similar PIGDO algorithms such as PIAdagrad and PIRMSprop.

Remark 4. In Reference [1], the authors used moment generating function to study the privacy loss and obtained its upper bound. From the proof in the full edition of this article, we find that the overall privacy loss makes use of the equivalence relation $\log (1+\gamma) \sim \gamma$. However, they cannot ensure $\gamma \rightarrow 0$, i.e., $\frac{q^{2} \lambda^{2}}{\sigma^{2}} \rightarrow 0$ always hold. Moreover, these three parameters $q, \sigma$, and $\lambda$ usually make $\frac{q^{2} \lambda^{2}}{\sigma^{2}}>1+\log \left(\frac{q^{2} \lambda^{2}}{\sigma^{2}}\right)$, which can result in the higher upper bound of privacy loss. Therefore, in our MMA method, we remain the overall privacy loss as $T \log \left(1+\frac{q^{2} \lambda^{2}}{\sigma^{2}}\right)$ and by this way, we eventually get a tighter bound shown in Theorem 3. Specifically, we choose $q=0.01, \sigma=2$ and $\delta=10^{-5}$, when epoch $E=400$, i.e., $T=40,000$, the privacy loss $\varepsilon$ of their moments accountant method is 10.2 , but the privacy loss of MMA is 6.8. It should be noted that under the other different parameters, we can get the similar result.

Remark 5. As for the parameter selection, compared with Reference [1], we give detailed calculation procedures for the range of $q, \sigma, \lambda$, and our expression of privacy loss is exact. However, in Reference [1], there are some unspecified constants $c_{1}$ and $c_{2}$ in their theorem, which brings trouble to calculate how much noise should we add for achieving a given $(\varepsilon, \delta)$-DP. Although the 
open source TensorFlow privacy repo related to their paper provides code to compute the amount of noise necessary for a given privacy level, it is still more complex than our exact expression. The key reason why we obtain the exact formula is that we utilize the Property 1 and Property 2 to eliminate the parameter $\lambda$. But in the proof of Reference [1], authors seem to give a converse inequality according to the tail bound. Therefore, they cannot eliminate the extra parameter $\lambda$, which makes the calculation of privacy loss rather complex.

\section{PERFORMANCE EVALUATION}

\subsection{Experimental Setup}

To evaluate our experiments, we perform three popular image classification tasks including MNIST handwritten digit recognition [6], CIFAR-10 image classification [26], and Fashion-MNIST fashion image classification [42]. MNIST includes 60,000 training images and 10,000 testing images. Each example of this dataset is a $28 \times 28$ pixel grayscale image. The non-private model of MNIST can reach the training/testing accuracy of $98.62 \% / 98.57 \%$ after 100 epochs, which shows that this model matches up to the latest model. CIFAR-10 dataset contains 50,000 training images and 10,000 testing images. Each example of this dataset is a $32 \times 32$ RGB image. The non-private baseline model for CIFAR-10 can reach the testing accuracy of $86 \%$ after 500 epochs. Our implementation is based on the TensorFlow implementation of DP-SGD in Reference [1]. For comparing, we choose the neural networks similar to theirs, that is, MNIST experiment only contains a fully connected layer with 1,000 hidden units, and CIFAR-10 experiment has two convolutional layers followed by a fully connected layer. The default lot size and clipping value are 600 and 4, respectively. Fashion-MNIST is a new dataset that can be seen as a replacement of the MNIST dataset, and it contains 60,000 training images and 10,000 testing images. Each example of this dataset is a $28 \times 28$ grayscale image. The total of 70,000 fashion products are divided into 10 categories, and each category has 7,000 images. The non-private model of Fashion-MNIST can achieve training/testing accuracy of $97.94 \% / 88.85 \%$ after 300 epochs. The neural network designed for Fashion-MNIST is the same as that of the MNIST dataset, and the default parameter setting is in accord with the above two datasets.

\subsection{Experimental Results}

Comparing three kinds of PIGDO algorithms: We first evaluate three kinds of PIGOD algorithms, i.e., PIAdagrad, PIAdam, and PIRMSprop on MNIST, CIFAR-10, and Fashion-MNIST datasets. Figure 3 shows the testing accuracy of these three differentially private optimization algorithms varies with the training process. Training accuracy result is similar and thus omitted. According to Figure 3, we can see that the model accuracies on CIFAR-10 and Fashion-MNIST datasets are lower than that on MNIST dataset, because the classification tasks of CIFAR-10 and Fashion-MNIST dataset are more difficult than that of MNIST dataset. Moreover, it can be observed that PIAdam algorithm always performs better than PIRMSprop and PIAdagrad algorithms on these three datasets. PIAdam algorithm has around 95.16\% accuracy on the MNIST dataset, 73.5\% accuracy on the CIFAR-10 dataset, and $83 \%$ accuracy on the Fashion-MNIST dataset. However, the testing accuracies of PIRMSprop and PIAdagrad on these three datasets are $94.4 \% / 68.93 \% / 81.99 \%$ and $93.47 \% / 67.34 \% / 79.99 \%$, respectively. The above results are in accord with the fact that the Adam algorithm utilizes the estimate of the first moment and the second moment of gradients to obtain the better adaptive gradient descent, but the Adagrad and RMSprop algorithms only use the estimate of the second moment of gradients. Therefore, in the following experiments, we choose the PIAdam algorithm to show the effectiveness of our PIGDO algorithm. Last, compared with the non-private version, Figure 3 also demonstrates that the accuracy of PIGDO algorithm degrades when the noise is added. 


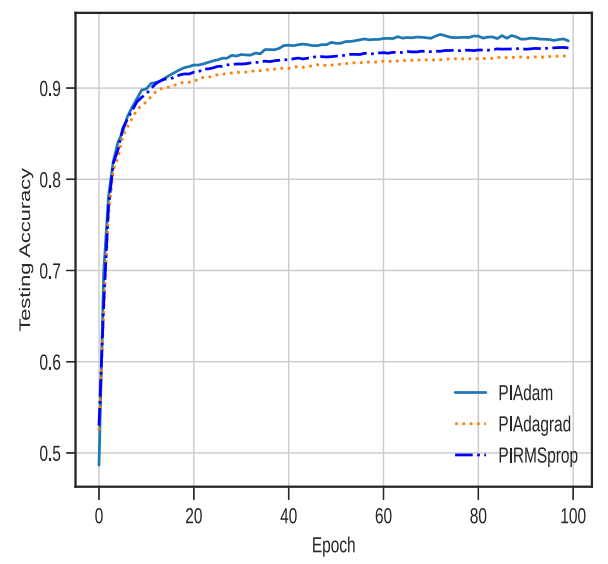

(a) MNIST

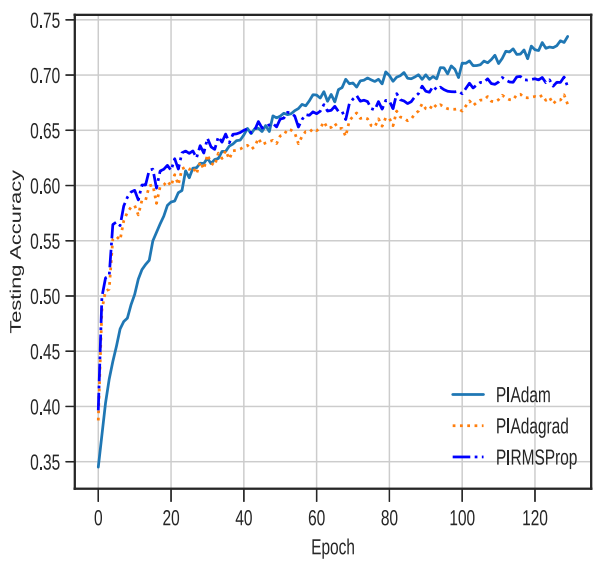

(b) CIFAR-10

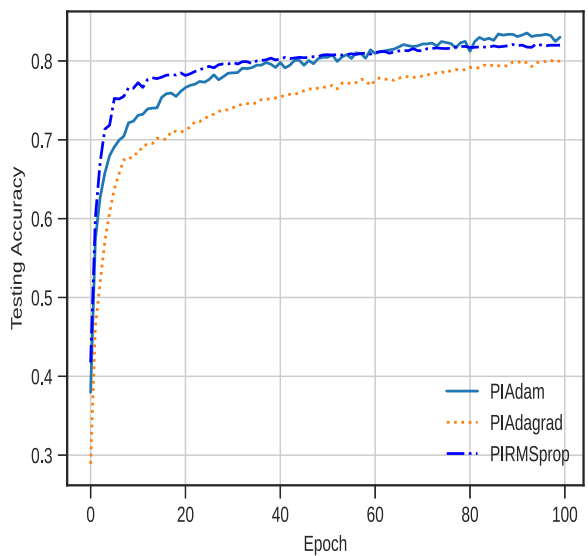

(c) Fashion-MNIST

Fig. 3. The testing accuracy of three kinds of PIGDO algorithms on MNIST, CIFAR-10, and Fashion-MNIST datasets, respectively.

To further illustrate the advantages of our GDO algorithm, we evaluate the PIAdam algorithm compared with several state-of-the-art algorithms that are listed as follows. These compared algorithms and our PIAdam algorithm are all evaluated on the same criteria.

- The ADPPL [14] is a differentially private algorithm for deep learning that adaptively injects noise into gradients based on the relevance between different features and the model output.

- The ADADP [43] is an adaptive and fast convergent algorithm that chooses the noise in the light of the sensitivity of each gradient and adds the sensitivity-dependent noise to achieve differential privacy.

- The EXP is one of the dynamic privacy budget allocation methods in Reference [44] that can be viewed as an adaptive noise addition method.

- The DPSGD [1] is the first work to combine the differential privacy with deep learning that adopts the gradient obfuscation to obtain the differential privacy protection.

Comparing Privacy Accounting: In regard to the differential privacy accounting, we compare our MMA method for our PIAdam algorithm with those privacy analysis methods used in the 


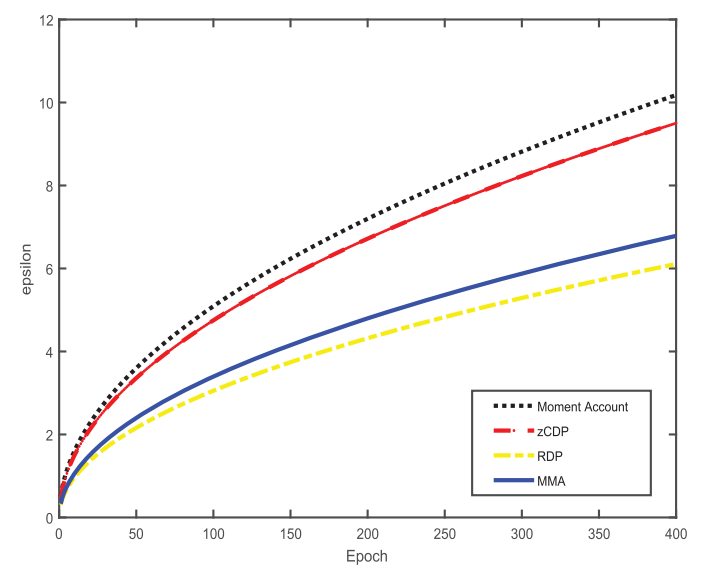

Fig. 4. The evolution of privacy loss epsilon changing with epoch for $\mathrm{q}=0.01, \sigma=2, \delta=10^{-5}$, using moments accountant, zCDP, RDP, and our MMA, respectively.

compared algorithms. We consider the moments accountant method in Reference [1] as the baseline. From Theorem 1 in their paper, we can derive that for deep learning with moments accountant method, when $\sigma=\frac{3 q \sqrt{T \log (1 / \delta)}}{\varepsilon}$, the DPSGD algorithm satisfies $(\epsilon, \delta)$-DP. For ADADP algorithm, Reference [43] adopted RDP method to account the privacy loss of their algorithm and showed the privacy loss is about half of the moments account method. zCDP used in EXP is essentially similar to moments accountant method and, therefore, the privacy budget of this algorithm is slightly smaller than the moments accountant method. For the ADPPL algorithm [14], the authors did not discuss the privacy accounting, and the noise addition was based on the Laplace mechanism, which is different from our Gaussian mechanism. At last, as to our privacy accounting method MMA, according to Theorem 3 in our work, we can guarantee that when $\sigma=\frac{2 q \log \frac{1}{\delta}}{\varepsilon \sqrt{\delta^{-\frac{1}{T}}-1}}$, the PIAdam satisfies $(\epsilon, \delta)$-DP.

Tocompare these methods more clearly, we set $q=0.01, \sigma=2$, and $\delta=10^{-5}$ as the default. It should be noted that for moment order $\lambda$, we choose $\lambda \leq 32$ similar to Reference [1], and if the corresponding privacy loss satisfies $\varepsilon>0.002 T / \lambda$, then our privacy loss has no relation to $\lambda$. We use the number of epochs to represent the running time, because we need many steps to train the deep learning model. Denoting the number of epochs as $E$, then the number of steps satisfies $T=E / q$. Figure 4 shows four curves that describe the evolution of privacy loss epsilon changing with epoch and they correspond to moments accountant, zCDP, RDP, and MMA, respectively. From Figure 4, it can be seen that our MMA method always has lower privacy loss than moments accountant and zCDP method. In addition, the privacy loss of our method grows more slowly than moments accountant and zCDP method. It means that for a given overall privacy budget, our method allows performing more epochs, which usually leads to achieving higher model accuracy. On the whole, our optimization method is more effective both on privacy preservation and model accuracy. In addition, although the privacy loss of our MMA method is slightly higher than RDP, the privacy accounting expression of MMA (i.e., the noise inequality in Theorem 1) is easier than that of RDP (i.e., Equation (7) in Reference [43]).

Results on MNIST: We compare our differentially private gradient descent optimization algorithm PIAdam with the ADPPL, ADADP, EXP, and DPSGD algorithm on MNIST dataset. In the training process, we set the lot size as 600 and the gradient norm bound as $C=4$. And we choose the default learning rate for PIAdam as 0.001 . 


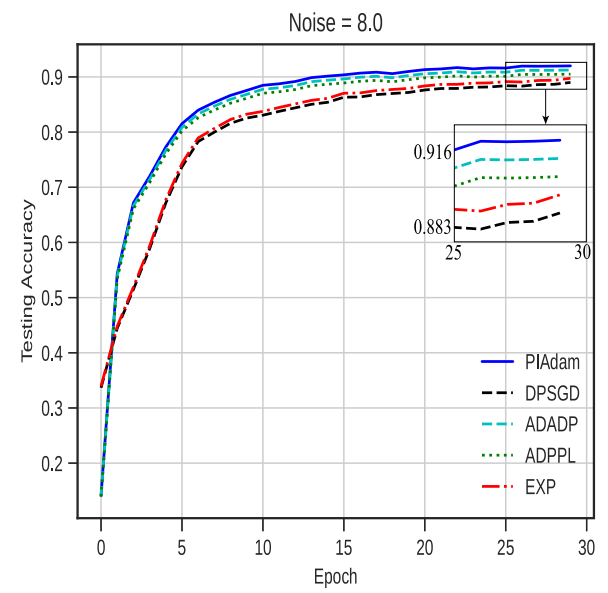

(a) Large noise $(\sigma=8)$

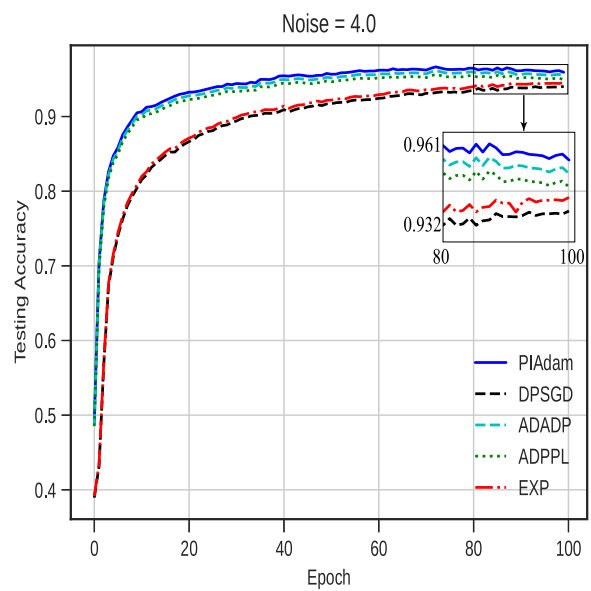

(b) Medium noise $(\sigma=4)$

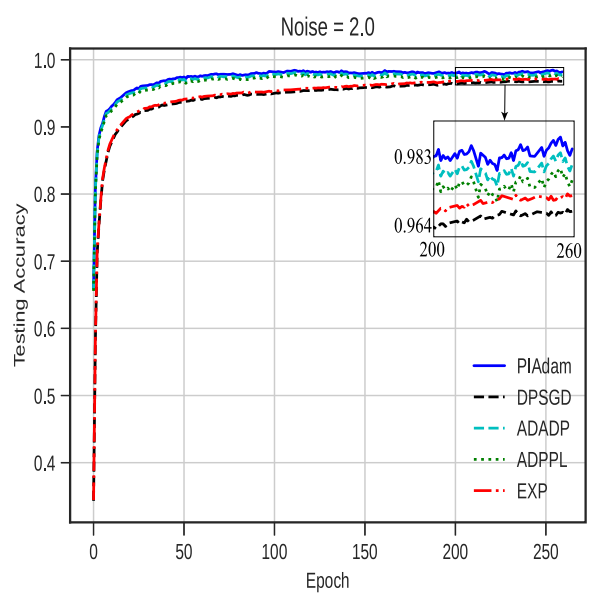

(c) Small noise $(\sigma=2)$

Fig. 5. Testing accuracy on MNIST dataset at different noise levels.

In terms of accuracy, Figure 5 gives the training results in three cases with different privacy levels: high privacy level corresponding to the large noise scale $(\sigma=8)$, medium privacy level corresponding to the moderate noise scale $(\sigma=4)$, and low privacy level corresponding to the small noise scale $(\sigma=2)$. In each plot, we give testing accuracies for PIAdam, ADADP, ADPPL, EXP, and DPSGD, and these testing accuracies change with the epoch. These results show that PIAdam surpasses or roughly equals with the other algorithms on testing accuracy at all levels. Specifically, when noise $=8$, testing accuracy is as high as $92.02 \%$ for PIAdam, which improves by $0.82 \%, 1.65 \%, 2.5 \%$, and $3.48 \%$ over ADADP (91.25\%), ADPPL (90.92\%), EXP (89.75\%), and DPSGD (88.91\%), respectively. Similarly, when noise $\sigma=4$, the testing accuracy of PIAdam achieves $95.93 \%$, which is close to the ADADP (95.43\%) and PIAdam still improves by $1.05 \%, 1.47 \%$, and $2 \%$ over ADPPL (94.94\%), EXP (94.54\%), and DPSGD (94\%), respectively. At noise=2, the testing accuracy of PIAdam obtains $98.21 \%$, which improves within $1 \%$ over ADADP, ADPPL, and EXP but still improves $1.44 \%$ over DPSGD $(96.81 \%)$. It can be demonstrated that our perturbed iterative gradient descent optimization algorithm can achieve better accuracy than the previous work. 


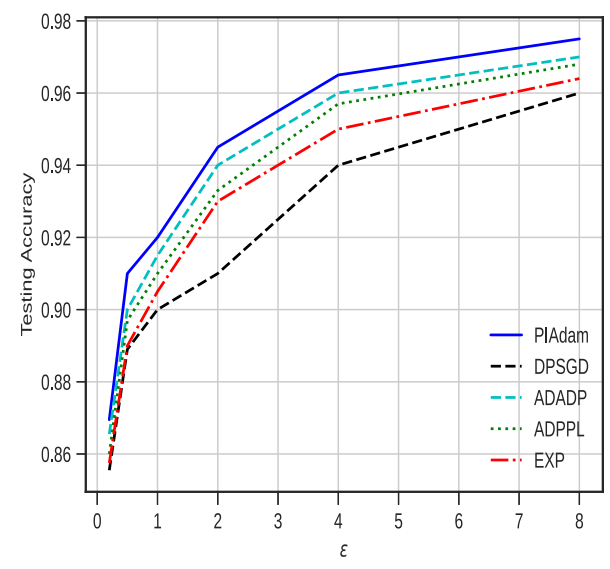

(a) the effect of $\varepsilon$

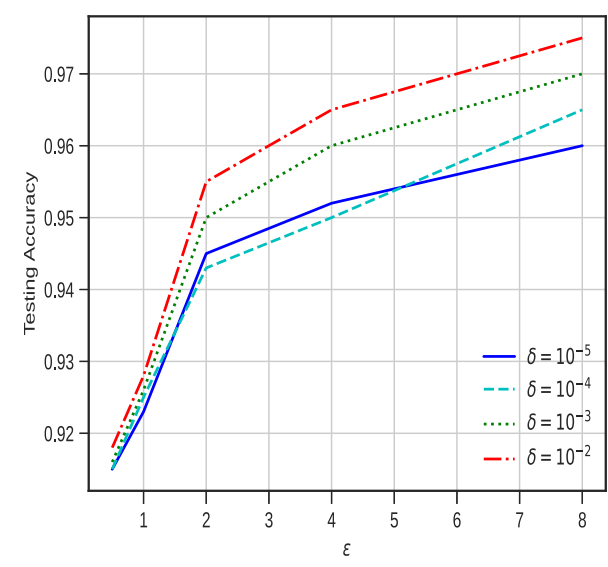

(b) the effect of $\delta$

Fig. 6. Effects of privacy parameter on accuracy over the MNIST dataset.

Additionally, we investigate the impact of noise on privacy preservation. Taking PIAdam as example, the testing accuracy can reach $98.2 \%$ at epoch $=260$ for noise $=2,95.93 \%$ at epoch $=100$ for noise $=4$, and $92.11 \%$ at epoch $=30$ for noise $=8$, respectively. Then, according to Figure 4 , for these three different epochs $=260,100$, and 30 , the corresponding privacy guarantee $\varepsilon$ is $5.47,3.39$, and 1.86, respectively. It indicates that the more noise there is, the better privacy preservation is achieved. In the end, comparing these DP algorithms with the non-private algorithms, we can draw a similar conclusion from the aforementioned discussion, which states that we achieve differential privacy guarantee at the expense of accuracy.

To illustrate the tradeoff between the model utility and the privacy protection, we plot Figure 6 to show the testing accuracy changing with the privacy parameter $\epsilon$ and $\delta$. Figure 6(a) exhibits the testing accuracy of five algorithms with different privacy budget $\epsilon$. The range of $\epsilon$ is from 0.2 to 8. It is obvious that our PIAdam algorithm has better model utility than DPSGD, ADADP, ADPPL, and EXP. Specifically, given privacy budget $\epsilon=0.5$, the testing accuracy of our PIAdam is 0.91 compared with $\operatorname{ADADP}(0.9), \operatorname{ADPPL}(0.897), \operatorname{EXP}(0.89)$, and DPSGD(0.889). When $\epsilon$ is large, e.g., $\epsilon=8$, our algorithm achieves 0.975 , which is close to the non-private testing accuracy. The curves in Figure 6(b) show the impact of relaxation factor $\delta$ on the testing accuracy for different privacy budget. $\delta$ varies from $10^{-5}$ to $10^{-2}$. According to Figure 6(b), we can see that the privacy budget $\epsilon$ is the major factor that affects the model accuracy, because $\delta$ has little impact on the classification accuracy of model regardless of the $\epsilon$ 's variation.

Effect of the parameters: The accuracy of classification task on MNIST dataset is affected by many hyperparameters such as the structure of neural network, the number of hidden units, and some training parameters consisting of lot size and learning rate. As for differentially private deep learning, there are some specific parameters, such as clipping value $C$ and noise scale $\sigma$. It is important to adjust these parameters carefully for optimal performance. To study the influence of these parameters, we tune them individually and keep the rest constant. We give the following default values: 1,000 hidden units, 600 lot size, clipping value $C=4$, and noise scale $\sigma=4$. Figure 7 shows the results of these varying parameters. In the following, we discuss the effect of these parameters on accuracy separately.

Number of hidden layer units: We change the number of hidden layer units in neural network from 200 to 1,600. From Figure 7(a), we can see that all the PIGDO algorithms are insensitive to 


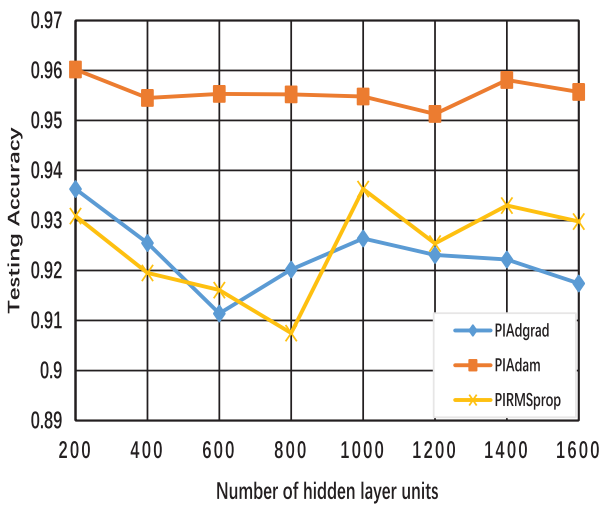

(a) Hidden Units

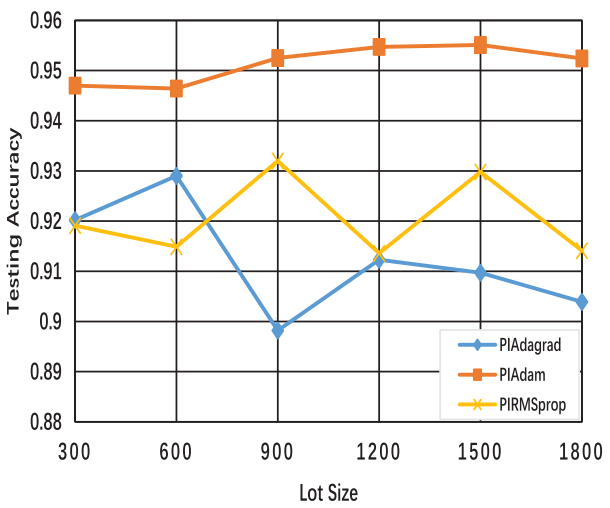

(c) Lot Size $L$

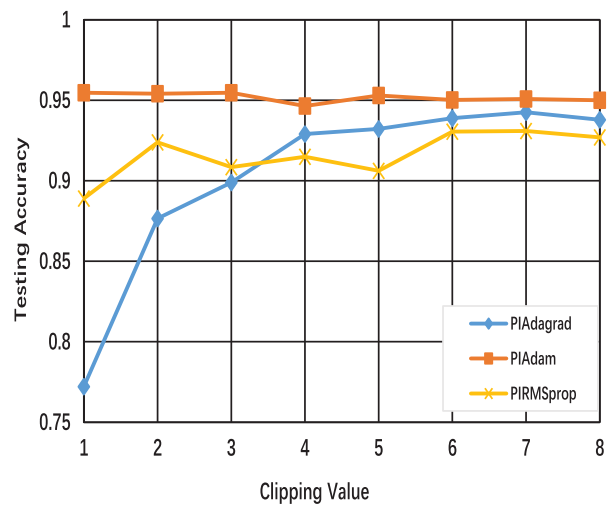

(b) Clipping Value $C$

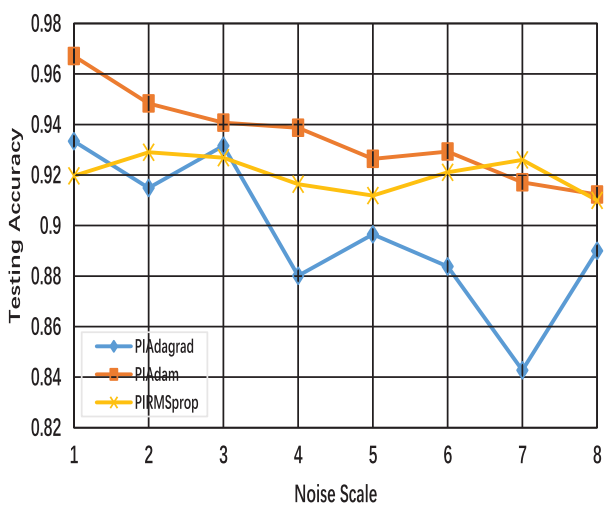

(d) Noise Level $\sigma$

Fig. 7. When only one parameter varies, testing accuracy on MNIST dataset vs. number of hidden layer unit, clipping value, lot size, and noise level, respectively.

the variation of network topology. This case is very different from the non-private training, which usually requires more units to fit the training dataset more easily. Moreover, as has been shown in Figure 7(a), even though increasing hidden units add the sensitivity of the gradient, which brings more noise into each step, we can see that more the number of hidden units does not reduce the accuracy of the training model. Specifically, with the variation of the number of hidden layer units, the change of testing accuracy is within $3 \%$ for all algorithms. This is consistent with the discussion of DP-SGD in Reference [1]. One possible reason is that deeper networks can tolerate more noise.

Clipping value: Gradient clipping brings two opposing effects in accuracy. On one hand, clipping is harmful to the unbiasedness of the gradient estimate, and when the clipping value is too small, the average of the clipping gradient may go to a very different direction from the true gradient. On the other hand, the larger clipping value leads to adding more noise to the gradients. Then, according to Figure 7(b), the testing accuracy of PIAdagrad increases as the clipping value becomes bigger. It indicates that PIAdagrad is more sensitive to the variation of gradient direction than the additive noise. However, for the testing accuracy of PIAdam and PIRMSprop algorithms, the impact of these two effects is equally important and, therefore, the testing accuracy of PIAdam and PIRMSprop algorithms changes a little with the variation of clipping value. 


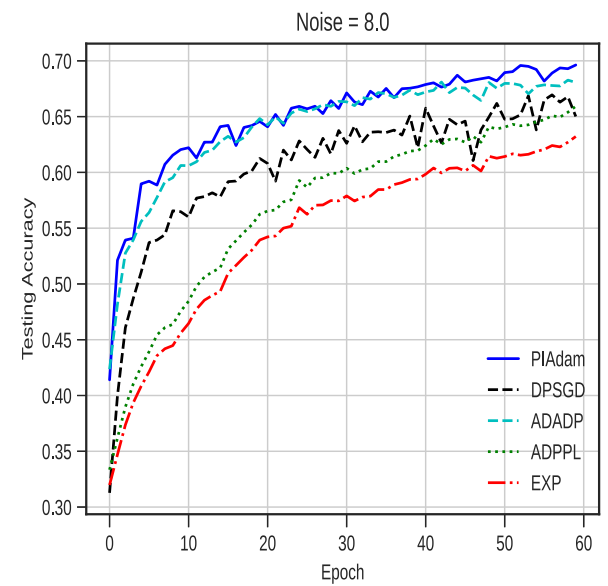

(a) Large noise $(\sigma=8)$

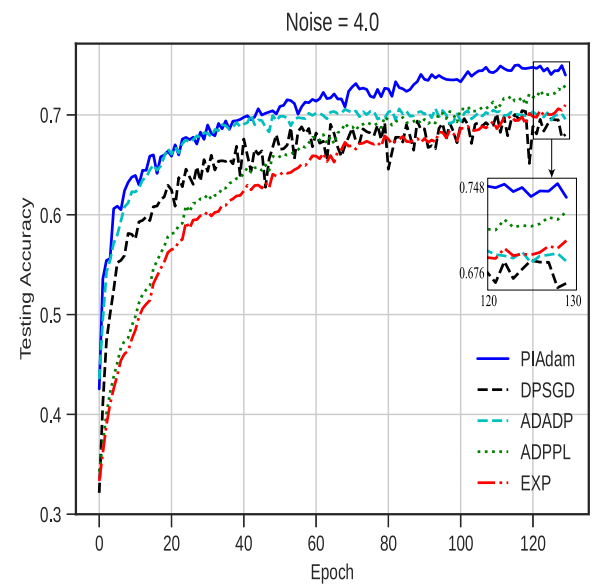

(b) Medium noise $(\sigma=4)$

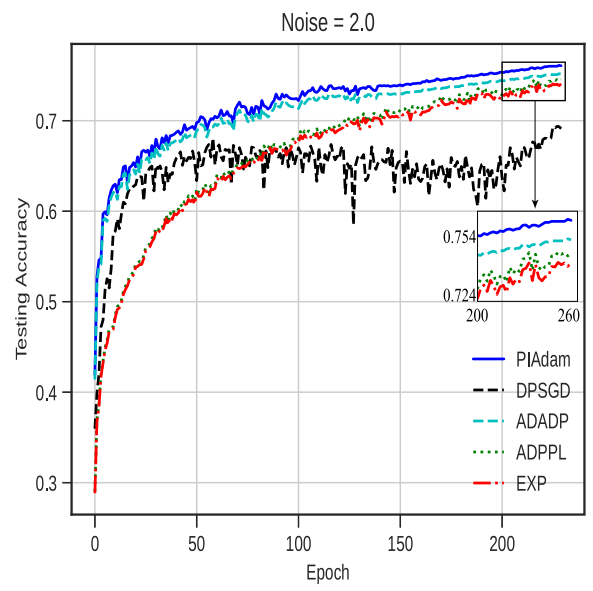

(c) Small noise $(\sigma=2)$

Fig. 8. Testing accuracy on CIFAR-10 dataset at different noise levels.

Lot size: From Figure 7(c), PIAdam algorithm achieves better testing accuracy than the PIAdagrad and PIRMSprop algorithms with the variation of lot size. The reason is that larger lot leads to less epoch, which usually causes the worse model accuracy. However, as has been discussed before, PIAdam algorithm has the better adaptive gradient descent, which only requires relatively less epoch to train a model. Therefore, it can still achieve high accuracy when the lot is large.

Noise level: By adding more noise, the per-step privacy loss is proportionally smaller, so we can run more epochs within a given cumulative privacy budget. The choice of noise level has a large impact on accuracy.

Results on CIFAR-10 and Fashion-MNIST: Even though it is more difficult to reach high accuracy on more complex datasets such as CIFAR-10 and Fashion-MNIST, we illustrate that our optimization algorithm still has the advantage over the previous method. Similar to the MNIST task, we compare our PIAdam algorithm with the other four algorithms on CIFAR-10 dataset. In our experiment, the default training parameter is 600 lot size, $\sigma=2$ and $C=4$. Figure 8 shows that on CIFAR-10 dataset, PIAdam always achieves better accuracy than the rest of the algorithms 


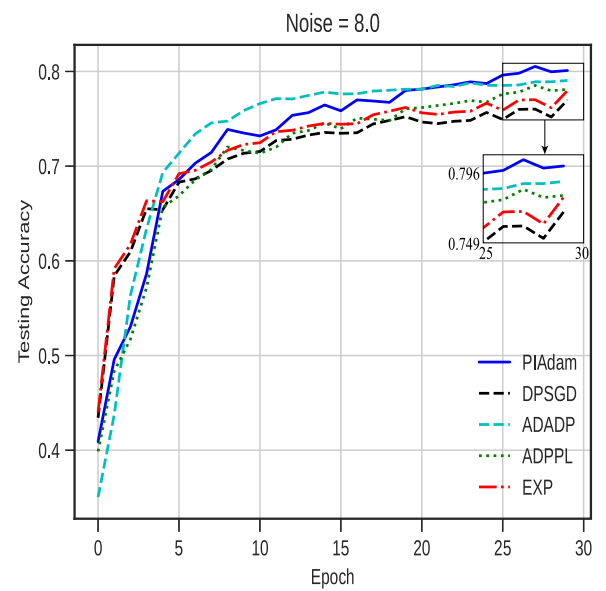

(a) Large noise $(\sigma=8)$

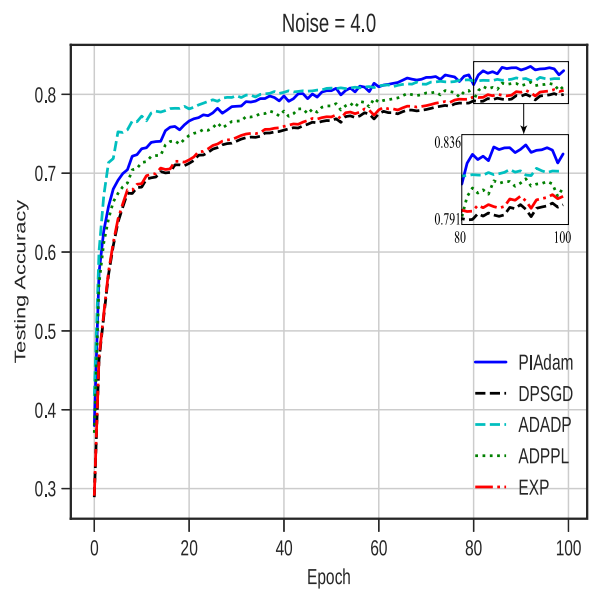

(b) Medium noise $(\sigma=4)$

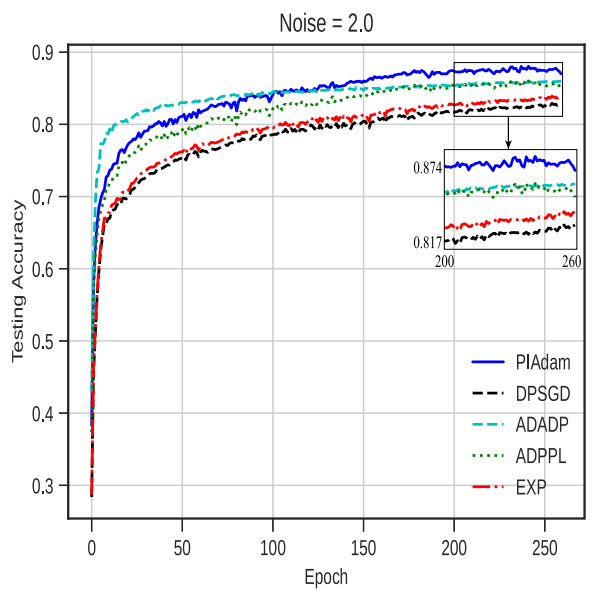

(c) Small noise $(\sigma=2)$

Fig. 9. Testing accuracy on Fashion-MNIST dataset at different noise levels.

at all the noise levels. Specifically, when noise $\sigma=8$, testing accuracy is as high as $69.63 \%$ for PIAdam, which improves by $2.3 \%, 5.6 \%, 10.14 \%$, and $7.1 \%$ over ADADP $(68.06 \%)$, ADPPL (65.93\%), EXP (63.22\%), and DPSGD (65.01\%), respectively. Similarly, when noise $\sigma=4$, the testing accuracy of PIAdam achieves $73.99 \%$, which is close to the ADPPL (73\%), and PIAdam still improves by $6.37 \%, 4.21 \%$, and $8.73 \%$ over ADADP (69.56\%), EXP (71\%), and DPSGD (68.05\%), respectively. At noise $=2$, the testing accuracy of PIAdam obtains $76.07 \%$, which improves within $2 \%$ over ADADP, ADPPL, and EXP but still improves $10.07 \%$ over DPSGD (69.11\%). It can be demonstrated that our perturbed iterative gradient descent optimization algorithm can achieve better accuracy than the previous work. In addition, we can see that on CIFAR-10 dataset, the testing accuracy of DPSGD varies dramatically but by using the above optimization methods, the improved algorithms including PIAdam achieve the relatively stable testing accuracy. In the end, similarly, we also do the experiments on the Fashion-MNIST dataset to further validate the general applicability of our differentially private algorithms. Consistent with the previous experimental setting, we also give the training results in three cases with different privacy levels: high privacy level corresponding to 
the large noise scale $(\sigma=8)$, medium privacy level corresponding to the moderate noise scale $(\sigma=4)$, and low privacy level corresponding to the small noise scale $(\sigma=2)$. Figure 9 shows that on Fashion-MNIST dataset, our PIGDO algorithm still surpasses the other latest algorithms at all noise levels.

\section{CONCLUSION AND FUTURE WORK}

In this article, we combine the differentially private gradient descent optimization algorithm with deep learning to achieve privacy preservation. Based on the proposed perturbed iterative gradient descent optimization (PIGDO) algorithm that integrates the gradient descent algorithm as an iterative component and then injects noise into the gradients computed by iterative GDO, we can perform the gradient perturbation for achieving the differential privacy. Compared with the state-of-the-art methods, our perturbed iterative gradient descent optimizations including PIAdam, PIRMSprop, and PIAdagrad succeed in the better model accuracy and training speed. Moreover, in the privacy analysis, to obtain a tighter bound of privacy loss, we propose a modified moments accountant (MMA) method to investigate the privacy accounting problem, and our expression of privacy loss is exact and easy to compute. More importantly, the MMA method can get the tighter bound of privacy loss compared with the other popular privacy accounting method. Our experiments on MNIST, CIFAR-10, and Fashion-MNIST datasets demonstrate the effectiveness of our optimization algorithm.

In the future, we prepare to extend our framework to the distributed scenario, which is more widely used in the big data era. For this challenging work, we aim to minimize the total privacy loss and achieve the satisfying tradeoff between utility and privacy preservation for each agent. However, different from the centralized deep learning with differential privacy, the existing noise addition method needs to be redesigned under the distributed framework and the information exchange between different agents affects the overall privacy budget. Therefore, it is an interesting but challenging future work. Besides, we want to combine the better gradient perturbation techniques like adaptive clipping with more efficient privacy accountant methods like concentrated differential privacy to improve the performance. It is crucial for differential-privacy-typed work to seek the elaborate privacy budget allocation method, which can significantly improve the data utility under the moderate privacy requirements. Adaptive gradient clipping can exactly be viewed as a potential direction to develop the satisfactory gradient perturbation method. Finally, concentrated differential privacy is a new analysis method about privacy loss, and it can be used to record the privacy loss more accurately. Overall, the combination of adaptive gradient clipping with concentrated differential privacy is a meaningful research topic.

\section{REFERENCES}

[1] Martin Abadi, Andy Chu, Ian Goodfellow, H. Brendan McMahan, Ilya Mironov, Kunal Talwar, and Li Zhang. 2016. Deep learning with differential privacy. In Proceedings of the ACM SIGSAC Conference on Computer and Communications Security. ACM, 308-318.

[2] Yoshinori Aono, Takuya Hayashi, Lihua Wang, Shiho Moriai, et al. 2017. Privacy-preserving deep learning via additively homomorphic encryption. IEEE Trans. Inf. Forens. Secur. 13, 5 (2017), 1333-1345.

[3] Alceu Bissoto, Eduardo Valle, and Sandra Avila. 2021. GAN-based data augmentation and anonymization for skinlesion analysis: A critical review. In Proceedings of the IEEE/CVF Conference on Computer Vision and Pattern Recognition. $1847-1856$.

[4] George E. Dahl, Dong Yu, Li Deng, and Alex Acero. 2011. Context-dependent pre-trained deep neural networks for large-vocabulary speech recognition. IEEE Trans. Audio, Speech Lang. Process. 20, 1 (2011), 30-42.

[5] Jorge Dávila-Chacón, Jindong Liu, and Stefan Wermter. 2018. Enhanced robot speech recognition using biomimetic binaural sound source localization. IEEE Trans. Neural Netw. Learn. Syst. 30, 1 (2018), 138-150.

[6] Li Deng. 2012. The MNIST database of handwritten digit images for machine learning research [best of the web]. IEEE Sig. Process. Mag. 29, 6 (2012), 141-142. 
[7] Xiaofeng Ding, Hongbiao Fang, Zhilin Zhang, Kim-Kwang R. Choo, and Hai Jin. 2020. Privacy-preserving feature extraction via adversarial training. IEEE Trans. Knowl. Data Eng. (2020), 1-1. DOI : https://doi.org/10.1109/TKDE.2020 2997604

[8] Cynthia Dwork, Frank McSherry, Kobbi Nissim, and Adam Smith. 2006. Calibrating noise to sensitivity in private data analysis. In Proceedings of the Theory of Cryptography Conference. Springer, 265-284.

[9] Cynthia Dwork and Aaron Roth. 2014. The algorithmic foundations of differential privacy. Found. Trends Theoret. Comput. Sci. 9, 3-4 (2014), 211-407.

[10] Cynthia Dwork, Guy N. Rothblum, and Salil Vadhan. 2010. Boosting and differential privacy. In Proceedings of the IEEE 51st Annual Symposium on Foundations of Computer Science. IEEE, 51-60.

[11] Matt Fredrikson, Somesh Jha, and Thomas Ristenpart. 2015. Model inversion attacks that exploit confidence information and basic countermeasures. In Proceedings of the ACM SIGSAC Conference on Computer and Communications Security. ACM, 1322-1333.

[12] Matthew Fredrikson, Eric Lantz, Somesh Jha, Simon Lin, David Page, and Thomas Ristenpart. 2014. Privacy in pharmacogenetics: An end-to-end case study of personalized Warfarin dosing. In Proceedings of the 23rd USENIX Security Symposium. 17-32.

[13] Craig Gentry. 2009. Fully homomorphic encryption using ideal lattices. In Proceedings of the 41st Annual ACM Symposium on Theory of Computing. 169-178.

[14] Maoguo Gong, Ke Pan, Yu Xie, A. Kai Qin, and Zedong Tang. 2020. Preserving differential privacy in deep neural networks with relevance-based adaptive noise imposition. Neural Netw. 125 (2020), 131-141.

[15] Kaiming He, Xiangyu Zhang, Shaoqing Ren, and Jian Sun. 2016. Deep residual learning for image recognition. In Proceedings of the IEEE Conference on Computer Vision and Pattern Recognition. IEEE, 770-778.

[16] Xixi Huang, Jian Guan, Bin Zhang, Shuhan Qi, Xuan Wang, and Qing Liao. 2019. Differentially private convolutional neural networks with adaptive gradient descent. In Proceedings of the IEEE 4th International Conference on Data Science in Cyberspace (DSC). IEEE, 642-648.

[17] Melvin Johnson, Mike Schuster, Quoc V. Le, et al. 2017. Google's multilingual neural machine translation system: Enabling zero-shot translation. Trans. Assoc. Computat. Ling. 5 (2017), 339-351.

[18] Diederik P. Kingma and Jimmy Ba. 2014. Adam: A method for stochastic optimization. arXiv preprint arXiv:1412.6980.

[19] Antti Koskela and Antti Honkela. 2020. Learning rate adaptation for differentially private learning. In Proceedings of the International Conference on Artificial Intelligence and Statistics. PMLR, 2465-2475.

[20] Jaewoo Lee and Daniel Kifer. 2018. Concentrated differentially private gradient descent with adaptive per-iteration privacy budget. In Proceedings of the 24th ACM SIGKDD International Conference on Knowledge Discovery \& Data Mining. ACM, 1656-1665.

[21] Jaewoo Lee and Daniel Kifer. 2020. Scaling up differentially private deep learning with fast per-example gradient clipping. arXiv preprint arXiv:2009.03106.

[22] Tiancheng Li, Ninghui Li, and Jian Zhang. 2009. Modeling and integrating background knowledge in data anonymization. In Proceedings of the IEEE 25th International Conference on Data Engineering. IEEE, 6-17.

[23] Yehida Lindell. 2005. Secure multiparty computation for privacy preserving data mining. In Encyclopedia of Data Warehousing and Mining. IGI Global, 1005-1009.

[24] Xiaoyu Liu, Shunda Pan, Qi Zhang, Yu-Gang Jiang, and Xuanjing Huang. 2019. Reformulating natural language queries using sequence-to-sequence models. Sci. China Inf. Sci. 62.

[25] Xu Ma, Fangguo Zhang, and Xiaofeng Chen. 2018. Privacy preserving multi-party computation delegation for deep learning in cloud computing. Inf. Sci. 459 (2018), 103-116.

[26] Ari Morcos, Haonan Yu, Michela Paganini, and Yuandong Tian. 2019. One ticket to win them all: Generalizing lottery ticket initializations across datasets and optimizers. In Proceedings of the International Conference on Advances in Neural Information Processing Systems. 4932-4942.

[27] Mahesh Chandra Mukkamala and Matthias Hein. 2017. Variants of RMSProp and Adagrad with logarithmic regret bounds. In Proceedings of the 34th International Conference on Machine Learning (ICML'17). 2545-2553.

[28] Ayahiko Niimi. 2018. Study on data anonymization for deep learning. In Proceedings of the International Conference on Industrial, Engineering and Other Applications of Applied Intelligent Systems. Springer, 762-767.

[29] NhatHai Phan, Yue Wang, Xintao Wu, and Dejing Dou. 2016. Differential privacy preservation for deep auto-encoders: An application of human behavior prediction. In Proceedings of the 30th AAAI Conference on Artificial Intelligence. 1309-1316.

[30] NhatHai Phan, Xintao Wu, Han Hu, and Dejing Dou. 2017. Adaptive laplace mechanism: Differential privacy preservation in deep learning. In Proceedings of the IEEE International Conference on Data Mining (ICDM). IEEE, 385-394.

[31] Théo Ryffel, David Pointcheval, Francis Bach, Edouard Dufour-Sans, and Romain Gay. 2019. Partially encrypted deep learning using functional encryption. Adv. Neural Inf. Process. Syst. 32 (2019), 4517-4528. 
[32] Andrey V. Savchenko. 2019. Probabilistic neural network with complex exponential activation functions in image recognition. IEEE Trans. Neural Netw. Learn. Syst. 31, 2 (2019), 651-660.

[33] Reza Shokri and Vitaly Shmatikov. 2015. Privacy-preserving deep learning. In Proceedings of the ACM SIGSAC Conference on Computer and Communications Security. ACM, 1310-1321.

[34] Reza Shokri, Marco Stronati, Congzheng Song, and Vitaly Shmatikov. 2017. Membership inference attacks against machine learning models. In Proceedings of the IEEE Symposium on Security and Privacy (SP). IEEE, 3-18.

[35] Congzheng Song, Thomas Ristenpart, and Vitaly Shmatikov. 2017. Machine learning models that remember too much. In Proceedings of the ACM SIGSAC Conference on Computer and Communications Security. ACM, 587-601.

[36] Shuang Song, Kamalika Chaudhuri, and Anand D. Sarwate. 2013. Stochastic gradient descent with differentially private updates. In Proceedings of the IEEE Global Conference on Signal and Information Processing. IEEE, 245-248.

[37] Zongkun Sun, Yinglong Wang, Minglei Shu, Ruixia Liu, and Huiqi Zhao. 2019. Differential privacy for data and model publishing of medical data. IEEE Access 7 (2019), 152103-152114.

[38] Tijmen Tieleman and Geoffrey Hinton. 2012. Lecture 6.5-rmsprop: Divide the gradient by a running average of its recent magnitude. COURSERA: Neural Netw. Mach. Learn. 4, 2 (2012), 26-31.

[39] Anh-Tu Tran, The-Dung Luong, Jessada Karnjana, and Van-Nam Huynh. 2021. An efficient approach for privacy preserving decentralized deep learning models based on secure multi-party computation. Neurocomputing 422 (2021), 245-262.

[40] Yu-Xiang Wang, Borja Balle, and Shiva Prasad Kasiviswanathan. 2019. Subsampled Rényi differential privacy and analytical moments accountant. In Proceedings of the 22nd International Conference on Artificial Intelligence and Statistics. PMLR, 1226-1235.

[41] L. Xiang, J. Yang, and B. Li. 2019. Differentially-private deep learning from an optimization perspective. In Proceedings of IEEE International Conference on Computer Communications (INFOCOM). IEEE, 559-567.

[42] Han Xiao, Kashif Rasul, and Roland Vollgraf. 2017. Fashion-MNIST: A novel image dataset for benchmarking machine learning algorithms. arXiv preprint arXiv:1708.07747.

[43] Zhiying Xu, Shuyu Shi, Alex X. Liu, Jun Zhao, and Lin Chen. 2020. An adaptive and fast convergent approach to differentially private deep learning. In Proceedings of IEEE International Conference on Computer Communications (INFOCOM). IEEE, 1867-1876.

[44] Lei Yu, Ling Liu, Calton Pu, Mehmet Emre Gursoy, and Stacey Truex. 2019. Differentially private model publishing for deep learning. In Proceedings of the IEEE Symposium on Security and Privacy (SP). IEEE, 332-349.

Received March 2021; revised June 2021; accepted October 2021 